TRANSACTIONS OF THE

AMERICAN MATHEMATICAL SOCIETY

Volume 354, Number 8, Pages 3117-3154

S 0002-9947(02)03007-6

Article electronically published on April 2, 2002

\title{
SEMILINEAR NEUMANN BOUNDARY VALUE PROBLEMS ON A RECTANGLE
}

\author{
JUNPING SHI
}

AbStRaCt. We consider a semilinear elliptic equation

$$
\Delta u+\lambda f(u)=0, \quad \mathbf{x} \in \Omega, \quad \frac{\partial u}{\partial n}=0, \quad \mathbf{x} \in \partial \Omega,
$$

where $\Omega$ is a rectangle $(0, a) \times(0, b)$ in $\mathbf{R}^{2}$. For balanced and unbalanced $f$, we obtain partial descriptions of global bifurcation diagrams in $(\lambda, u)$ space. In particular, we rigorously prove the existence of secondary bifurcation branches from the semi-trivial solutions, which is called dimension-breaking bifurcation. We also study the asymptotic behavior of the monotone solutions when $\lambda \rightarrow$ $\infty$. The results can be applied to the Allen-Cahn equation and some equations arising from mathematical biology.

\section{INTRODUCTION}

We consider the stationary solutions of a semilinear parabolic equation with homogeneous Neumann boundary condition:

$$
\begin{cases}u_{t}=\Delta u+\lambda f(u), & t>0, \mathbf{x} \in \Omega, \\ \frac{\partial u}{\partial n}=0, & t>0, \mathbf{x} \in \partial \Omega \\ u(0, \mathbf{x})=u_{0}(\mathbf{x}), & \mathbf{x} \in \Omega,\end{cases}
$$

where $\lambda$ is a positive parameter, and $\Omega$ is a bounded smooth domain in $\mathbf{R}^{n}$ with $n \geq 1$. The stationary solutions are those independent of the time variable $\left(u_{t}=0\right)$, and the set of the stationary solutions is very important in studying the dynamic behavior of solutions of (1.1). However, in general it is hard to get a complete description of the stationary solution set, especially when the dimension of the domain $\Omega$ is more than 1 . In this paper, we study the set of the stationary solutions of (1.1) for $\Omega$ being a rectangle in $\mathbf{R}^{2}$, and we rigourously show that the bifurcation diagrams for two-dimensional problems have much richer structures than the wellstudied ordinary differential equation (1.2). Moreover we are able to classify the profiles of all monotone solutions in certain cases when the parameter $\lambda \rightarrow \infty$.

Received by the editors April 17, 2001.

2000 Mathematics Subject Classification. Primary 35J25, 35B32; Secondary 35J60, 34C11.

Key words and phrases. Semilinear elliptic equations, secondary bifurcations, global bifurcation diagrams, asymptotic behavior of solutions. 


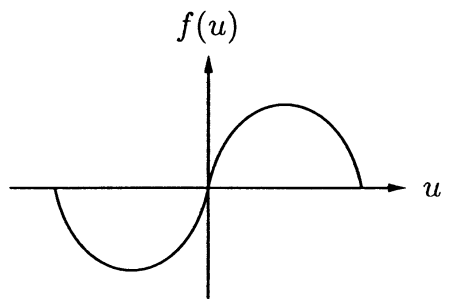

FiguRE 1. Balanced $f(u)$

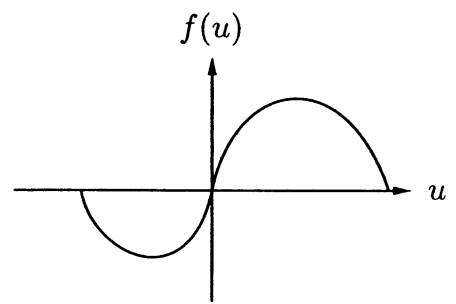

FIGURE 2. Unbalanced $f(u)$

To describe our results, let us start with a simple equation in one-dimensional space:

$$
\left\{\begin{array}{l}
u^{\prime \prime}+\lambda f(u)=0, \quad x \in(0, a), \\
u^{\prime}(0)=u^{\prime}(a)=0 .
\end{array}\right.
$$

We assume that $f$ is a sufficiently smooth function. If $f(c)=0$ for some $c \in \mathbf{R}$, then $u(x) \equiv c$ is a trivial solution of (1.2). If $u$ is a nonconstant solution, we call it a nontrivial solution. Under very general conditions on $f$, it is known that the set of nontrivial solutions $\Sigma$ consists of curves, and on each curve the solutions have fixed nodal mode. A detailed description of the set $\Sigma$ will be given in Section 2 . Here we assume that there exists a solution curve of (1.2)

$$
\Sigma_{1}=\{(\lambda(s), u(s, x)): s \in(m, M)\}
$$

for some $m, M>0$, and $s$ is a parameter. We can connect the solutions of (1.2) to the solutions of the equation on a rectangle:

$$
\begin{cases}\Delta u+\lambda f(u)=0, & (x, y) \in \Omega \equiv(0, a) \times(0, b), \\ \frac{\partial u}{\partial n}=0, & (x, y) \in \partial \Omega .\end{cases}
$$

In fact, we could easily obtain a solution of (1.4) by defining $v(x, y)=u(x)$, where $u$ is a solution of (1.2). We call such $v(x, y)$ a semi-trivial solution, which is only $x$-dependent. In particular, if we have a solution curve $\Sigma_{1}$ of (1.2), then we obtain a solution curve of (1.4),

$$
\widetilde{\Sigma_{1}}=\left\{(\lambda(s), v(s, x, y)): v(s, x, y)=u(s, x),(\lambda(s), u(s, x)) \in \Sigma_{1}\right\} .
$$

In this paper, we discuss several problems related to the solution curve $\widetilde{\Sigma_{1}}$, and it leads to a better understanding of the solution set of (1.4).

In our study, we consider two types of nonlinearities $f(u)$. The prototypes are

$$
\begin{aligned}
& \text { (A) } f(u)=u-u^{3}=-u(u-1)(u+1) \quad \text { (balanced, see Fig. 1); } \\
& \text { (B1) } f(u)=-u+u^{p}, u>0, p>1 \quad \text { (unbalanced); } \\
& \text { (B2) } f(u)=-u(u-a)(u+1), a>1 \quad \text { (unbalanced, see Fig. 2). }
\end{aligned}
$$

The nonlinearity (A) is called balanced since $\int_{-1}^{1} f(u) d u=0$, where -1 and 1 are the two stable zeros of $f(u)$. (B2) is unbalanced since $\int_{-1}^{a} f(u) d u>0$, and (B1) is also unbalanced since $\int_{0}^{K} f(u) d u>0$ for sufficiently large $K>0$, while 0 is the only stable zero of $f(u)$. Here a zero $u_{0}$ of $f(u)$ is stable if and only if $f^{\prime}\left(u_{0}\right)<0$, in the sense that $u_{0}$ is a stable equilibrium point of $u^{\prime}=f(u)$. 


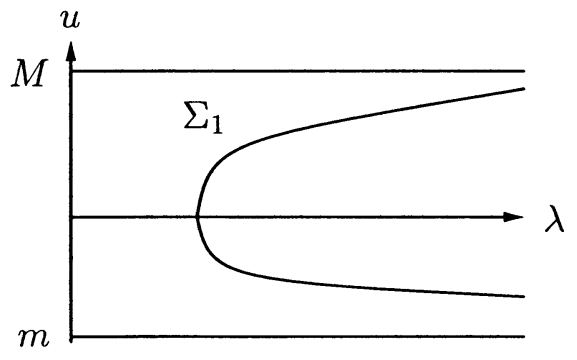

FiguRE 3. Solution curve for both balanced and unbalanced $f$

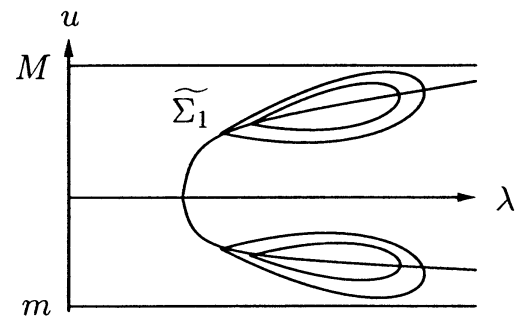

Figure 4. Mushroom for balanced $f$

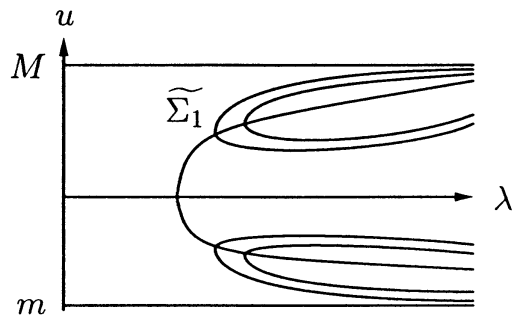

FiguRE 5. Tree for unbalanced $f$

Now we assume that $\Sigma_{1}$ is a curve of monotone decreasing solutions. For the one-dimensional problem (1.2),$\Sigma_{1}$ for balanced and unbalanced $f$ are qualitatively the same (see Fig. 3). In fact, $\lambda^{\prime}(s)>0$ on the upper branch, and the Morse index of a solution on $\Sigma_{1}$ is always 1. The Morse index $M(u)$ of a solution $(\lambda, u)$ to (1.4) or (1.2) is the number of negative eigenvalues $\mu_{k}$ of

$$
\begin{cases}\Delta \phi+\lambda f^{\prime}(u) \phi=-\mu \phi, & \mathbf{x} \in \Omega, \\ \frac{\partial \phi}{\partial n}=0, & \mathbf{x} \in \partial \Omega,\end{cases}
$$

where $\Omega$ is either $(0, a) \times(0, b)$ or $(0, a)$ respectively. However, we will show that the magnitudes of the principal eigenvalue $\mu_{1}(\lambda(s)$ ) for (A) and (B) are different, and that makes the Morse indices of the semi-trivial solutions $v(s)$ on $\widetilde{\Sigma_{1}}$ different for (A) and (B). As $\lambda(s) \rightarrow \infty, M(v(s)) \rightarrow 1$ for (A) and $M(v(s)) \rightarrow \infty$ for (B). Along $\widetilde{\Sigma_{1}}$, when $M(v(s))$ changes from $k$ to $k+1$, a secondary bifurcation occurs at $(\lambda(s), v(s))$. We will show that a bifurcation theorem of Crandall and Rabinowitz [CR can be applied here, and a pitchfork bifurcation occurs. This can also be viewed as a symmetry breaking along a branch of symmetric solutions (semi-trivial solutions). Because of properties of $M(v(s))$, there is a sequence of infinitely many bifurcation points along $\widetilde{\Sigma_{1}}$ for (B), but only a finite number of bifurcation points for (A). So the solution set around $\widetilde{\Sigma_{1}}$ looks like a "tree" (Fig. 5) with infinitely many branches for (B), and it looks like a "mushroom" (Fig. 4) for (A). This type of secondary bifurcations can also be called dimension breaking bifurcations.

For balanced $f$, (1.2) was first studied by Chafee and Infant [CI], and the corresponding parabolic equation (1.1) (the Allen-Cahn equation) received a lot of 
attention in the last two decades; several aspects, like slow motions of the interfaces and the existence of equilibrium solutions with interfaces, have been studied. (See [AFK], $\mathrm{AFS}],[\mathrm{BK}],[\mathrm{CP}],[\mathrm{DFP}],[\mathrm{FH} 2],[\mathrm{KS}], \mathrm{PT}]$.$) Most works assume$ $\lambda \rightarrow \infty$. Recently, Ghoussoub and Gui [GG] proved that any solution $u$ of

$$
\Delta u+f(u)=0, \quad \mathbf{x}=(x, y) \in \mathbf{R}^{2},
$$

satisfying $\partial u / \partial x<0$ must be a form of $u(x, y)=u(a x+b y)$ for some $a, b \in \mathbf{R}$. In particular, this implies that when $f$ is balanced, any monotone solution of (1.7) must be $U_{1}(a x+b y)$, where $U_{1}$ is the unique monotone decreasing solution of $u^{\prime \prime}+f(u)=0$ in $\mathbf{R}$. (See 2.23).) The proof of this result uses some techniques developed by Berestycki, Caffarelli and Nirenberg in their work on the qualitative properties of elliptic equations in unbounded domains $[\mathrm{BCN}]$, and it answers a conjecture by De Giorgi in the two-dimensional case. (See [GG] and [BCN] for more details.)

Our bifurcation result draws an interesting comparison between (1.4) and (1.7). First it shows that when $\lambda$ is not so large, (1.4) has monotone solutions on the secondary branches with nonflat interface (an interface is the level set $\{u=0\}$ ). On the other hand, when $\lambda$ is large, the monotone solutions must have flat interfaces as in the case of (1.7), except in the case of a square. In fact, we obtain a complete classification of the monotone solutions of (1.4) when $\lambda$ is sufficiently large for balanced $f$, which is interesting by itself (a monotone solution $u(x, y)$ of (1.4) is such that $u_{x}$ and $u_{y}$ do not change sign in $\Omega$ ):

Suppose that $u$ is a solution of (1.4) such that $u_{x} \leq 0$ and $u_{y} \leq 0$ for $(x, y) \in \bar{\Omega}$. If $f$ is balanced, then there exists $\bar{\lambda}>0$ such that for $\lambda>\bar{\lambda}$, u must be one of the following:

- a constant solution;

- a semi-trivial solution such that $u_{x} \equiv 0, u_{y}<0$ in $\Omega$;

- a semi-trivial solution such that $u_{y} \equiv 0, u_{x}<0$ in $\Omega$;

- only when $\Omega$ is a square, a solution whose interface intersects two opposite vertices at $45^{\circ}$.

This result is also the reason why the secondary bifurcation curves are indeed bounded, so the "mushrooms" will not go to infinity. It is no surprise that our proof of the above result uses the theorem of [GG], in the blowup arguments as $\lambda \rightarrow \infty$. The proof also use the geometric theory of the dynamics of the scalar Allen-Cahn equation and elliptic estimates. On the other hand, the solutions with diagonal nodal lines which occur only in the square case are related to the only other known solution to (1.7): the saddle solution. The existence and uniqueness of the saddle solution for $f(u)$ being odd and $f(u) / u$ decreasing was proved by Dang, Fife and Peletier [DFP]. In a companion paper [S4] to the current one, the author proves the existence and uniqueness of the saddle solution when $f$ is a balanced nonlinearity, via proving the existence of diagonal interface solutions of (1.4).

The uniqueness or exact multiplicity of the solutions to a semilinear elliptic equation is usually a rather difficult question. Problems of a similar nature have been studied extensively for Dirichlet boundary conditions; see, for example, D1], D2, [D3], OS1, OS2], [S1], S2], [S3], SWj and references therein. In general, Dirichlet boundary conditions are much more rigid than Neumann boundary conditions, and one consequence is that problems with a Neumann boundary condition tend to have a lot more solutions. In the Neumann case, in contrast to the huge amount 
of work on the small diffusion situation, very little work has been done on the structure of global branches of solutions, and all previous results seem to be on the one-dimensional problem (1.2) (see [SmW], $[\mathrm{Sc}],[\mathrm{Kor}]$ ). Our result here is an exact multiplicity result for a real partial differential equation with Neumann boundary condition in higher dimensional spaces.

In the study of some semilinear problems, numerical analysis and informal arguments indicate that there are a lot of mushroom type bifurcations (bounded branches of solutions), e.g., the perturbed Gelfand problem, and the Sel'kov model from combustion theory (see, for example, [MS]). But little is rigorously known for the existence of such mushroom type bifurcations. Here we show that it could happen for the balanced nonlinearity when the rectangle is narrow in one direction. On the other hand, there are no previous results on the secondary bifurcation for autonomous equations like (1.4), as we show in this paper. The secondary bifurcation, like a tree structure for a non-autonomous equation with balanced $f$, was studied by Fusco and Hale FH1.

For the Allen-Cahn equation, Alikakos, Fusco and Kowalczyk AFK proved the existence/nonexistence and stability of transition layer solutions to (1.4) when the domain is a rectangle with two additional parts attached. Recently Maier-Paape and Miller [MM numerically described the solution set of (1.4) with balanced $f(\mathrm{~A})$ and unbalanced $f$ (B2) when $\Omega$ is a square. There are no secondary bifurcations observed on the semi-trivial branches. For the balanced case, we should point out that "mushrooms" exist only when $a / b$ or $b / a$ is large (see Section 4 for details). So in the case of square, it is likely that there are no secondary bifurcations from the semi-trivial branches. But in the unbalanced case, there are always infinite many secondary bifurcations.

For the unbalanced nonlinearity $f(u)=-u+u^{p}$, the elliptic equation (1.4) (with general bounded domain $\Omega$ ) arises from the studies of pattern formation in morphogenesis and chemotaxis. (See Ni [N].) From the "tree" diagram, Fig. 5, all secondary bifurcation branches are unbounded in the $\lambda$ direction, and when $\lambda \rightarrow \infty$, it seems that the solution is near 0 in most areas of $\Omega$, but have "spikes" at certain locations. We call these solutions spike layer solutions. In recent years, the spike layer solutions have been the subjects of numerous studies; see, for example, [BDS, [BS], [BFi], [BFu, DY1, DY2], G], GW1, GW2, GWW], [Kow, [L], [LNT], [NT2, [NT3], W], WW1, WW2 and references therein. But to confirm rigourously that these solutions are spike layer solutions, we need a better understanding of the solutions of $\Delta u+f(u)=0$ in $\mathbf{R}^{2}$. Due to the limit of the length of the paper, the work in that direction will appear in another paper [S5, and we prove there that the solutions in the secondary branches are indeed spike layer solutions. We also remark that for a general bounded domain $\Omega$, the tree structure of the solution set may not persist, and the bifurcation structure of the solution set will be more complicated.

A semi-trivial solution on the primary branch of the tree also concentrates into a small area when $\lambda \rightarrow \infty$, but instead of a spike layer, a one-dimensional sharp boundary layer (or interior layer for non-monotone solutions) exists. We call such a solution a 1-layer solution. From bifurcation analysis, a 1-layer solution is more unstable than a spike layer solution, and its energy level is also much higher than that of a spike layer solution. The bifurcation diagram suggests that for the corresponding parabolic equation, there would be connecting orbits between 1-layer solutions 
and spike layer solutions with spikes on the 1-dimensional layer. In his survey paper [N], Ni asked about the existence of such $k$-layer solutions for general domains. From the information we obtain here, such solutions will have the following characterization: (i) the energy $E(u)=O\left(\lambda^{-1 / 2}\right)$, (ii) the Morse index $M(u)=O\left(\lambda^{1 / 2}\right)$, and (iii) the set of the maximum points converges to a one-dimensional manifold. Even from our special case, we should notice that the set of the maximum points may not be a manifold itself, since the secondary bifurcation solutions with many small spikes also fit the above description when they are near bifurcation points.

The semi-trivial stationary solutions of the reaction-diffusion system

$$
\begin{cases}\tau u_{t}=\varepsilon \Delta u+\varepsilon^{-1} f(u, v), & t>0, \mathbf{x} \in \Omega \\ v_{t}=D \Delta v+g(u, v), & t>0, \mathbf{x} \in \Omega \\ \frac{\partial u}{\partial n}=\frac{\partial v}{\partial n}=0, & t>0, \mathbf{x} \in \partial \Omega \\ u(0, \mathbf{x})=u_{0}(\mathbf{x}), v(0, \mathbf{x})=v_{0}(\mathbf{x}), & \mathbf{x} \in \Omega,\end{cases}
$$

where $\Omega=(0, a) \times(0, b)$, were considered by Taniguchi and Nishiura [TN], [T], where the stability of the semi-trivial solutions and local bifurcations from the curve of the semi-trivial solutions were studied. While the analysis of the linearized problems is much harder for a system, our results for the single equation are more complete. We prove that there is a sequence of bifurcations from the curve of semi-trivial solutions, and we also obtain global bifurcation results and classify the asymptotic profiles of the solutions.

The Cahn-Hilliard equation

$$
\begin{cases}u_{t}=\varepsilon^{2} \Delta(-\Delta u-f(u)), & t>0, \mathbf{x} \in \Omega, \\ \frac{\partial u}{\partial n}=\frac{\partial \Delta u}{\partial n}=0, & t>0, \mathbf{x} \in \partial \Omega, \\ u(0, \mathbf{x})=u_{0}(\mathbf{x}), & \mathbf{x} \in \Omega,\end{cases}
$$

is closely related to Allen-Cahn equation. The stationary solutions of (1.9) when $\Omega=(0,1)$ was studied in CGS] and [BFi, and the stationary solutions of the Cahn-Hilliard equation in higher dimensions were studied in [BDS, FKMW], Ki], [WW1], WW2]. In [FKMW] and [Ki], Cahn-Hilliard equations on a rectangle were also studied via bifurcation approaches. But their emphases are different from ours here: they both consider bifurcations from higher eigenvalues, thus obtaining solutions with more complicated nodal structure, while we consider bifurcations from the semi-trivial solutions. Also they did not consider secondary bifurcations. Our approach here essentially also works for the Cahn-Hilliard equation, and we will report similar results on the Cahn-Hilliard equation in a forthcoming paper.

In Section 2, we consider the one-dimensional problem (1.2), and the results there are mostly known. In Section 3, we consider the primary bifurcations from the trivial solutions, and in Section 4, we study the secondary bifurcations from the semi-trivial solutions. In Section 5, the global properties of the solution branches are considered. In Sections 6, we prove our classification of the monotone decreasing solutions for balanced $f$ when $\lambda \rightarrow \infty$. In the paper, we often use $C$ or $C_{i}(i \in \mathbf{N})$ for positive constants, which may be different in different places. For a linear operator $L, N(L)$ is the null space of $L$ and $R(L)$ is the range space of $L . \partial u / \partial n$ or $\partial_{n}$ is the outer normal derivative of $u$. 
Acknowledgement. The author would like to thank Professors Peter Bates, WeiMing Ni and Xuefeng Wang for their helpful conversations when this manuscript was prepared, and he also would like to thank the referee for some helpful comments. The first version of the paper was prepared when the author was visiting Tulane University, and he would like to thank them for their hospitality. The work of the author is also partially supported by a grant from the College of William and Mary.

\section{One-Dimensional Problem}

Consider

$$
\left\{\begin{array}{l}
u^{\prime \prime}+\lambda f(u)=0, \quad x \in(0, a) \\
u^{\prime}(0)=u^{\prime}(a)=0
\end{array}\right.
$$

where $f \in C^{1}(\mathbf{R})$. In this section, we summarize some key facts about the solution set of (2.1). Most results here are more or less known to experts in the field, but there is no suitable reference for all of them. Other results on (2.1) can be found in $[\mathrm{SmW}],[\mathrm{Sc}$ and [Kor]. (2.1) can be converted to a first-order system:

$$
u^{\prime}=v, v^{\prime}=-\lambda f(u) .
$$

In fact, a solution $u$ of (2.1) is equivalent to a solution $(u, v)$ of (2.2) with boundary condition $v(0)=v(a)=0$. If $\left(\lambda_{0}, u_{0}(x)\right)$ is a monotone decreasing solution of (2.1), then $\left(u_{0}, u_{0}^{\prime}\right)$ is the lower half of a periodic orbit on the phase portrait of (2.2) with $\lambda=\lambda_{0}$, and for any $n \in \mathbf{N},(\lambda, u)=\left(n^{2} \lambda_{0}, u_{0}(n x)\right)$ is also a solution, which can be viewed as the same periodic orbit wrapping around for $n / 2$ periods with faster time. So any nonmonotone solution is just a rescaled reflective and/or periodic extension of a monotone solution. On the other hand, if $u_{0}(x)$ is a monotone increasing solution of (2.1), then $u_{0}(a-x)$ is a monotone decreasing solution. Therefore the monotone decreasing solutions determine all other solutions of (2.1).

Next we show that all monotone decreasing solutions can be parameterized by the initial value of the solution $u(0)$. In fact, it is standard (see $[\mathrm{Sc}],[\mathrm{SmW}$ ) to derive a time-mapping formula from the equation: if $(\lambda, u)$ is a monotone decreasing solution of (2.1), $u(0)=s$, and $u(a)=g(s)$, then

$$
\sqrt{\lambda}=\frac{1}{\sqrt{2} a} \int_{g(s)}^{s} \frac{d u}{\sqrt{F(s)-F(u)}} \equiv T(s),
$$

where $F(u)=\int_{0}^{u} f(t) d t$, and $g(s)$ is determined by $F(s)=F(g(s))$ and $F(u)<$ $F(s)$ for all $u \in(g(s), s)$. Therefore for each $s \in \mathbf{R}$ satisfying $f(s)>0$, there is at most one $\lambda>0$ such that (2.1) has a monotone decreasing solution $(\lambda, u)$ such that $u(0)=s, \lambda=\lambda(s)=[T(s)]^{2}$, which is uniquely determined by $s$, and the admissible set for $s$ is

$$
\begin{aligned}
S= & \{s \in \mathbf{R}: f(s)>0, \text { there exists a } g(s)<s \text { such that } \\
& F(s)=F(g(s)) \text { and } F(u)<F(s) \text { for all } u \in(g(s), s)\} .
\end{aligned}
$$

It is easy to see that $f(g(s))<0$, and $\lambda(s)$ is a smooth function if $f$ is sufficiently smooth. Summarizing these discussions, we have the following result:

Proposition 2.1. Suppose that $f \in C^{1}(\mathbf{R})$, and $\Sigma$ is the set of all nontrivial solutions of (2.1). Then

$$
\Sigma=\bigcup_{k=1}^{\infty} \Sigma_{k}^{ \pm}
$$




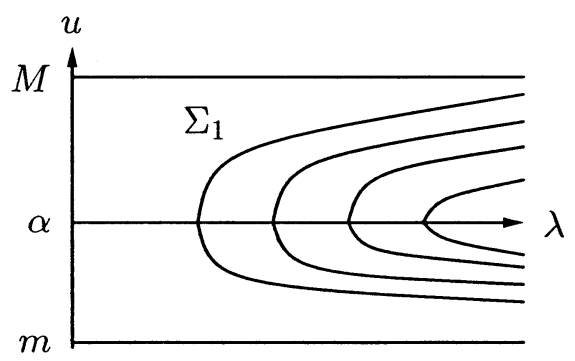

Figure 6. Solution set of (2.1)

where

$$
\begin{aligned}
& \Sigma_{k}^{+}=\left\{\left(\lambda_{k}(s), u_{k}(s, x)\right): s \in S\right\}, \\
& \Sigma_{k}^{-}=\left\{\left(\lambda_{k}(s), u_{k}(s, x)\right): g^{-1}(s) \in S\right\} .
\end{aligned}
$$

For all solutions, $u_{k}(s, 0)=s, \lambda_{1}(s)=[T(s)]^{2}$, where $T(s)$ is given by (2.3) for $s \in S, \lambda_{1}(s)=\lambda_{1}\left(g^{-1}(s)\right)$ for $s \in g(S)$, and $\lambda_{k}(s)=k^{2} \lambda_{1}(s)$ for $k>1$ (see Fig. $6)$.

The graphs of $\lambda=\lambda_{k}(s)$ exhaust all possible solutions of (2.1) on the bifurcation diagram, but from Proposition 2.1, the graph of $\lambda=\lambda_{1}(s)$ is sufficient to depict the whole picture. Also, even for $\lambda_{1}(s)$, there is a one-to-one correspondence between $\Sigma_{1}^{+}$and $\Sigma_{1}^{-}$in the sense that if $(\lambda, u(x)) \in \Sigma_{1}^{+}$, then $(\lambda, u(a-x)) \in \Sigma_{1}^{-}$. In the following, we will use $\Sigma_{k}$ for $\Sigma_{k}^{+} \cup \Sigma_{k}^{-}$.

Next we consider the stability of the solutions. Let $(\lambda, u)$ be a solution of (2.1). Consider an eigenvalue problem,

$$
\phi^{\prime \prime}+\lambda f^{\prime}(u) \phi=-\mu \phi, \quad x \in(0, a), \quad \phi^{\prime}(0)=\phi^{\prime}(a)=0 .
$$

(2.7) has a sequence of eigenvalues $\left\{\mu_{i}\right\}_{i=1}^{\infty}$ such that $\mu_{i}<\mu_{i+1}$ and $\lim _{i \rightarrow \infty} \mu_{i}=\infty$. If the principal eigenvalue $\mu_{1}(u)>0$, then $u$ is called stable, otherwise unstable. A solution $u$ is degenerate if $\mu_{i}=0$ for some $i \in \mathbf{N}$, otherwise nondegenerate. If a solution $u$ is unstable, then its Morse index $M(u)$ is the number of negative eigenvalues of (2.7). If $\left(\lambda\left(s_{0}\right), u\left(s_{0}, x\right)\right)$ is a degenerate solution, then $s_{0}$ is a critical point on the solution curve, i.e. $\lambda^{\prime}\left(s_{0}\right)=0$. In fact, if we differentiate (2.1) with respect to $s$, then $\frac{\partial u}{\partial s}\left(s_{0}, x\right)$ is an eigenfunction for (2.7) with $\mu=0$. If $\lambda^{\prime \prime}\left(s_{0}\right) \neq 0$, then $\left(\lambda\left(s_{0}\right), u\left(s_{0}, x\right)\right)$ is a turning point on the solution curve.

The basic result on the stability of a solution to (2.1) is that any nontrivial solution is unstable. For later application we recall the higher dimensional version of this result by Casten, Holland $\mathrm{CaH}$ ] and Matano [M1]:

Theorem 2.2. Suppose that $f \in C^{1}(\mathbf{R})$, and $\Omega$ is a bounded convex Lipschitz domain in $\mathbf{R}^{n}$ for $n \geq 1$. If $(\lambda, u)$ is a nonconstant solution of

$$
\Delta u+\lambda f(u)=0, \quad \mathbf{x} \in \Omega, \quad \frac{\partial u}{\partial n}=0, \quad \mathbf{x} \in \partial \Omega,
$$

then

$$
\Delta \phi+\lambda f^{\prime}(u) \phi=-\mu \phi, \quad \mathbf{x} \in \Omega, \quad \frac{\partial \phi}{\partial n}=0, \quad \mathbf{x} \in \partial \Omega,
$$

has at least one negative eigenvalue. 
In fact, for the one-dimensional case, we have a more precise result:

Lemma 2.3. Let $(\lambda(s), u(s, \cdot)) \in \Sigma_{1}^{+}, s \in S$. Then $M(u(s, \cdot))=1$ if $\lambda^{\prime}(s) \geq 0$ and $M(u(s, \cdot))=2$ if $\lambda^{\prime}(s)<0$.

Proof. We consider the solution $w(s, x)$ of the linearized equation

$$
w^{\prime \prime}+\lambda(s) f^{\prime}(u(s, x)) w=0, \quad x \in(0, a), \quad w^{\prime}(0)=0, w(0)=1 .
$$

We claim that

1. $w(s, \cdot)$ has exactly one zero in $(0, a), w(s, a)<0$, and

2. $w_{x}(s, a) \leq 0$ if and only if $M(u(s, \cdot))=1$, and $w_{x}(s, a)>0$ if and only if $M(u(s, \cdot))=2$.

Since $u_{x}$ satisfies

$$
u_{x}^{\prime \prime}+\lambda f^{\prime}(u(s, x)) u_{x}=0, \quad x \in(0, a), \quad u_{x}(s, 0)=0, u_{x}(s, a)=0,
$$

$w(s, \cdot)$ satisfies the same equation as in (2.9), and $u_{x}(s, x)<0$ for all $x \in(0, a)$, it follows that $w(s, \cdot)$ has exactly one zero in $(0,1)$ by the Sturm comparison lemma, and $w(s, 1)<0$. In particular, if $w_{x}(s, a)=0$, then $w(s, \cdot)$ is a solution of (2.7) with $\mu=0$. Since $w(s, \cdot)$ changes sign exactly once, then $w(s, \cdot)$ is an eigenfunction corresponding to $\mu_{2}$. Thus $\mu_{2}=0$ and $M(u(s, \cdot))=1$. This proves the lemma when $\lambda^{\prime}(s)=0$.

For the case of $\lambda^{\prime}(s) \neq 0$, let $\left(\mu_{k}, \phi_{k}\right)$ be the $k$-th eigenpair of 2.7). First we show that $\mu_{1}<0$. In fact, from the variational characterization, $\phi_{1}$ must be of one sign. On the other hand, if $\mu_{1} \geq 0$, then by the Sturm comparison lemma, $\phi_{1}$ must have a zero in $(0, a)$, by comparing $\phi_{1}$ and $u_{x}$. Thus $\mu_{1}<0$. Next we assume $\mu_{2}>0$. From the properties of the eigenfunctions, we know that $\phi_{2}$ has exactly one zero in $(0, a)$, and we assume $\phi_{2}(0)>0$; the unique zero of $\phi_{2}$ is $r_{2} \in(0, a)$. $w(s, \cdot)$ also has a unique zero, which is denoted by $r_{1}$. By comparing $\phi_{2}$ and $w$, using $\mu_{2}>0$, we obtain $r_{1}>r_{2}$. By integrating the equations of $w$ and $\phi_{2}$ over $\left(r_{1}, a\right)$, we get

$$
-\phi_{2}(a) w_{x}(s, a)+\phi_{2}\left(r_{1}\right) w_{x}\left(s, r_{1}\right)=-\mu_{2} \int_{r_{1}}^{a} w(s, x) \phi_{2}(x) d x
$$

then $w_{x}(s, a)<0$ since $\phi_{2}(x)<0$, and $w(s, x) \leq 0$ for $x \in\left[r_{1}, a\right], w_{x}\left(s, r_{1}\right)<0$. Similarly we can show that $w_{x}(s, a)>0$ if $\mu_{2}<0$.

Now we show that $\operatorname{sign}\left(\lambda^{\prime}(s)\right)=-\operatorname{sign}\left(w_{x}(s, a)\right)$ when $\lambda^{\prime}(s) \neq 0$. First, $u_{s}(s, x)$ satisfies

$$
\left\{\begin{array}{l}
u_{s}^{\prime \prime}+\lambda(s) f^{\prime}(u(s, x)) u_{s}+\lambda^{\prime}(s) f(u(s, x))=0, \quad x \in(0, a) \\
\left(u_{s}\right)_{x}(s, 0)=0,\left(u_{s}\right)_{x}(s, a)=0
\end{array}\right.
$$

and by differentiating $u(s, 0)=s$ and $u(s, a)=g(s)$ with respect to $s$, we obtain $u_{s}(s, 0)=1$ and $u_{s}(s, a)=g^{\prime}(s)$. Multiplying (2.8) by $u_{s}$, 2.11) by $w(s, \cdot)$, subtracting and integrating over $(0,1)$, we obtain

$$
-u_{s}(s, a) w_{x}(s, a)+\lambda^{\prime}(s) \int_{0}^{a} f(u(s, x)) w(s, x) d x=0 .
$$

We need to determine $\int_{0}^{a} f(u(s, x)) w(s, x) d x$. Let $v(x)=x u_{x}(s, x)$. Then it is easy to check that $v$ satisfies

$$
\left\{\begin{array}{l}
v^{\prime \prime}+\lambda(s) f^{\prime}(u(s, x)) v+2 \lambda(s) f(u(s, x))=0, \quad x \in(0, a), \\
v(s, 0)=0, v(s, a)=0,
\end{array}\right.
$$


and $v_{x}(s, 0)=0, v_{x}(s, a)=u_{x x}(s, a)=-\lambda f(g(s))$. Multiplying (2.8) by $v$, (2.13) by $w(s, \cdot)$, subtracting and integrating over $(0, a)$, we obtain

$$
w(s, a) v_{x}(s, a)+2 \lambda(s) \int_{0}^{a} f(u(s, x)) w(s, x) d x=0 .
$$

Combining 2.12) and 2.14), we have

$$
w(s, a) f(g(s)) \lambda^{\prime}(s)=2 g^{\prime}(s) w_{x}(s, a) .
$$

Since $F(s)=F(g(s))$, we have $f(s)=f(g(s)) g^{\prime}(s)$; then $g^{\prime}(s)<0$, since $u_{x x}(s, 0)<$ 0 and $u_{x x}(s, a)>0$. And from the proof above, $w(s, a)<0$. Thus $\operatorname{sign}\left(\lambda^{\prime}(s)\right)=$ $-\operatorname{sign}\left(w_{x}(s, a)\right)$.

Remark 2.4. The proof of Lemma 2.3 can be extended to prove that if $(\lambda(s), u(s, \cdot))$ $\in \Sigma_{k}^{+}, s \in S$, then $M(u(s, \cdot))=k$ if $\lambda^{\prime}(s) \geq 0$ and $M(u(s, \cdot))=k+1$ if $\lambda^{\prime}(s)<0$. For the case of $\Sigma_{k}^{-}$, it is easy to see that $M(u(s, \cdot))=k$ if $\lambda^{\prime}(s) \leq 0$ and $M(u(s, \cdot))=k+1$ if $\lambda^{\prime}(s)>0$. This type of proofs for Dirichlet problems (one dimension or radially symmetric solutions on ball) was also used in Ouyang and Shi OS2, Shi and Wang SWj], and Shi [S2].

So far we have treated (2.1) for a general $f$. For some $f$, the set $S$ can be empty or quite complicated. From now on we restrict our attention to a special class of $f$. We assume that $f$ satisfies

(f1) $f \in C^{2}([m, M])$ for $-\infty \leq m<M \leq \infty$, and there exists $\alpha \in(m, M)$ such that $f(\alpha)=0, f^{\prime}(\alpha) \geq 0, f(u)<0$ for $u \in(m, \alpha)$ and $f(u)>0$ for $u \in(\alpha, M)$;

(f2) $\int_{\alpha}^{M} f(u) d u \geq \int_{\alpha}^{m} f(u) d u$;

(f3) if $m>-\infty$ or $M<\infty$, then $f(m)=0$ or $f(M)=0$, respectively.

Note that we must consider $f$ which changes sign, since $\int_{\Omega} f(u(\mathbf{x})) d \mathbf{x}=0$ if $u$ is a solution of the Neumann boundary value problem (1.2) or (1.4). So in (f1), we consider $f$ which changes sign only once. (f2) is only for definiteness, and we can also consider the case when $\geq$ is replaced by $\leq$. In the remaining part of this section, we only consider the solutions of (2.1) with $u(0) \in(m, M)$, i.e., we restrict $S$ to $S \cap(m, M)$. Let $\left(\lambda_{k}, \phi_{k}\right)$ be the $k$-th eigenpair of

$$
\phi^{\prime \prime}+\lambda \phi=0, \quad x \in(0, a), \quad \phi^{\prime}(0)=\phi^{\prime}(a)=0 .
$$

It is easy to see that $\lambda_{0}=0, \phi_{0}=1$, and $\lambda_{k}=\frac{k^{2} \pi^{2}}{a^{2}}, \phi_{k}(x)=\cos \left(\frac{k \pi x}{a}\right)$ for $k \geq 1$. Now we have an existence result of $\Sigma_{1}$ for (2.1).

Theorem 2.5. Suppose that $f$ satisfies (f1), (f2) and (f3). In addition, assume that

$$
\lim _{u \rightarrow \pm \infty} \frac{f(u)}{u}=f_{\infty} \in[0, \infty]
$$

if $(m, M)=(-\infty, \infty)$.

1. If $f^{\prime}(\alpha)>0$, then $\lambda_{*}=\lambda_{1} / f^{\prime}(\alpha)$ is a bifurcation point, $\Sigma_{1}$ bifurcates from $(\lambda, u)=\left(\lambda_{*}, \alpha\right) ; S=\left(m, g^{-1}(m)\right) \backslash\{\alpha\}$, and

$$
\lim _{s \rightarrow \alpha} \lambda_{1}(s)=\lambda_{*}, \quad \lim _{s \rightarrow m^{+}} \lambda_{1}(s)=\lim _{s \rightarrow g^{-1}(m)^{-}} \lambda_{1}(s)=\lambda_{\infty},
$$




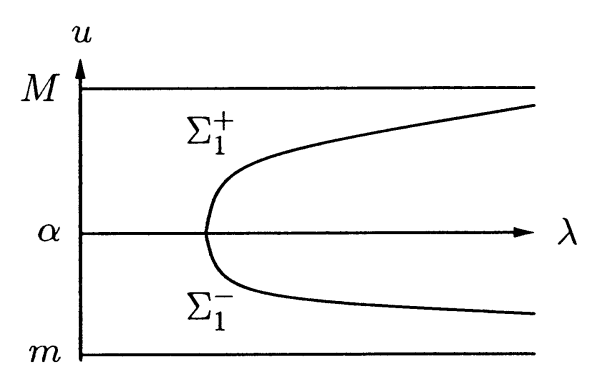

Figure 7. Bifurcation diagram for $f^{\prime}(\alpha)>0$

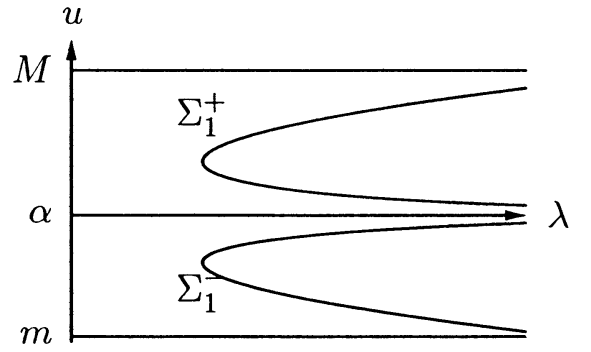

FIGURE 8. Bifurcation diagram for $f^{\prime}(\alpha)=0$

where $\lambda_{\infty}=\infty$ if $m>-\infty$ or $M<\infty$, and $\lambda_{\infty}=\lambda_{1} / f_{\infty}$ if $(m, M)=$ $(-\infty, \infty)$ (see Fig. 7).

2. If $f^{\prime}(\alpha)=0$, then $S=\left(m, g^{-1}(m)\right) \backslash\{\alpha\}$, and

$$
\lim _{s \rightarrow \alpha} \lambda_{1}(s)=\infty, \quad \lim _{s \rightarrow m^{+}} \lambda_{1}(s)=\lim _{s \rightarrow g^{-1}(m)^{-}} \lambda_{1}(s)=\lambda_{\infty}
$$

(see Fig. 8).

Proof. All statements can be obtained from estimating the time-mapping in (2.3). Here we apply bifurcation from the simple eigenvalue result to get $\Sigma_{1}$ locally at $\left(\lambda_{*}, \alpha\right)$ when $f^{\prime}(\alpha)>0 .(\lambda, u)=(\lambda, \alpha)$ is a line of trivial solutions of (2.1). Define $F(\lambda, u)=u^{\prime \prime}+\lambda f(u)$, where $\lambda>0, u \in X=\left\{u \in C^{2}([0, a]): u^{\prime}(0)=u^{\prime}(a)=0\right\}$. At $\left(\lambda_{*}, \alpha\right)$, the kernel $N\left(F_{u}\left(\lambda_{*}, \alpha\right)\right)=\operatorname{span}\{w\}, w(x)=\cos (\pi x / a)$, which is one dimensional; the range space $R\left(F_{u}\left(\lambda_{*}, \alpha\right)\right)=\left\{v \in C^{0}([0, a]): \int_{0}^{a} v(x) w(x) d x=\right.$ $0\}$, which is codimension 1; finally, $F_{\lambda u}\left(\lambda_{*}, \alpha\right) w=f^{\prime}(\alpha) w \notin R\left(F_{u}\left(\lambda_{*}, \alpha\right)\right)$, since $f^{\prime}(\alpha) \int_{0}^{a} w^{2}(x) d x \neq 0$. Hence we can apply the result of Crandall and Rabinowitz [CR] then the solution set of (2.1) near $(\lambda, u)=\left(\lambda_{*}, \alpha\right)$ consists of two parts: the line of constant solutions $\{(\lambda, \alpha)\}$ and a curve $\{(\lambda(s), u(s, x))\},\left|s-s_{0}\right| \leq \delta$, with $\left(\lambda\left(s_{0}\right), u\left(s_{0}, x\right)\right)=\left(\lambda_{*}, \alpha\right)$ and $u_{s}\left(s_{0}, x\right)=w(x)$. The solution $u(s, x)$ is monotone decreasing with respect to $x$ for $s>s_{0}$, since $u(s, x)=\alpha+\left(s-s_{0}\right) \cos (\pi x / a)+$ $o\left(\left|s-s_{0}\right|\right)$, and $u(s, x)$ is monotone increasing for $s<s_{0}$. From Proposition 2.1, the curve $(\lambda(s), u(s, x))$ is identical to $\Sigma_{1}$, and we can choose $s$ as $u(s, 0)$ and $s_{0}=\alpha$. $\Sigma_{1}$ can be extended for $s>\alpha$ as long as $g(s)$ is well defined. By (f2), we can extend $\Sigma_{1}^{-}$up to $s \in(m, \alpha)$, and correspondingly, $\Sigma_{1}^{+}$can be extended to $s \in\left(\alpha, g^{-1}(m)\right)$.

Next we prove the limit of $\lambda_{1}(s)$ is $\infty$ as $s \rightarrow \alpha$ when $f^{\prime}(\alpha)=0$. We apply (2.3). Since $f^{\prime}(\alpha)=0$, then for any $\eta>0$ there exists $\delta>0$ such that $|f(u)| \leq 2 \eta|u-\alpha|$ for $|u-\alpha| \leq \delta$; thus, $F(u) \leq F(\alpha)+\eta(u-\alpha)^{2}$ for $|u-\alpha| \leq \delta$. From the continuity of $g(s)$, there exists $\delta_{1} \in(0, \delta)$ such that for $0<u \leq \delta_{1}$ we have $-\delta \leq g(u)<0$. From (2.3) , for $s \in\left(0, \delta_{1}\right)$ we have

$$
\begin{aligned}
\sqrt{\lambda(s)} & =\frac{1}{\sqrt{2} a} \int_{g(s)}^{s} \frac{d u}{\sqrt{F(s)-F(u)}} \geq \frac{1}{\sqrt{2} a} \int_{g(s)}^{s} \frac{d u}{\sqrt{F(s)-F(\alpha)}} \\
& \geq \frac{s-g(s)}{\sqrt{2 \eta} a(s-\alpha)} \geq \frac{1}{\sqrt{2 \eta} a} .
\end{aligned}
$$

Since $\eta$ can be arbitrarily small, then $\lim _{s \rightarrow \alpha} \lambda_{1}(s)=\infty$.

Finally, we prove the asymptotic behavior of $\lambda_{1}(s)$. When one of $m$ and $M$ is finite, all the solutions are bounded, but $\Sigma_{1}$ is unbounded in $\mathbf{R}^{+} \times X$; so it has to 
be unbounded in the $\lambda$ direction, and $\lim _{s \rightarrow m^{+}} \lambda_{1}(s)=\infty$. For the proof in the case of $(m, M)=\mathbf{R}$, we refer to Theorem 3.5 of [S3], where the estimates of the time-mapping $\lambda_{1}(s)$ are given, and they are sufficient for the result here. See also OS2 for more detailed explanation on $f_{\infty}, \lambda_{\infty}$ and their impact on the bifurcation diagrams.

In the following discussion, we assume that $f$ satisfies (f1), (f2) and (f3). In addition we assume $f$ satisfies

(f4) $m>-\infty, f^{\prime}(m)<0$ and $f^{\prime}(\alpha)>0$.

Then $\Sigma_{1}$ must bifurcate from $\left(\lambda_{*}, \alpha\right)$; it is bounded by $u=m$ and $u=g^{-1}(m)$, and unbounded in the $\lambda$-direction, $\lim _{s \rightarrow m} \lambda_{1}(s)=\infty$. The asymptotic behavior of $\Sigma_{1}=\left\{\left(\lambda_{1}(s), u_{1}(s, x)\right)\right\}$ will play an important role in the bifurcation analysis, as will the pattern of the solutions in a rectangle. As we will see in Section 4, another important factor is $\mu_{1}(s)$, the principal eigenvalue of (2.7) for $u_{1}(s, \cdot)$. From Theorem [2.2, we know that $\mu_{1}(s)$ is always negative, but a more precise estimate will be needed. To state our result, we need to distinguish two different kinds of nonlinearities $f$ :

(f5a) $g^{-1}(m)=M, f(M)=0, f^{\prime}(M)<0, F(m)=F(M)$;

(f5b) $g^{-1}(m)<M, F(m)<F(M)$.

If $f$ satisfies (f1)-(f4) and (f5a), there is a heteroclinic orbit connecting the equilibrium points $(m, 0)$ and $(M, 0)$ on the phase portrait of (2.2). Because $F(m)=$ $F(M)$, we call $f$ a balanced nonlinearity. $f(u)=u-u^{3}$ is an example of a balanced function with $m=-1$ and $M=1$. If $f$ satisfies (f1)-(f4) and (f5b) (the unbalanced case), there is a homoclinic orbit starting from and terminating at $(m, 0)$, also going through $\left(g^{-1}(m), 0\right)$. That is the primary reason that we have different results for these two cases. The function $f(u)=-u+u^{p}, u>0, p>1$, is unbalanced with $m=0$ and $M=\infty$, while $f(u)=-u(u-a)(u+1), a>1$, is also unbalanced with $m=-1$ and $M=a$. Equation (2.1) with any of these functions as $f$ has a bifurcation diagram like Fig. 7 , but $\mu_{1}(s)$ is different, as shown in the following result:

Proposition 2.6. Suppose that $f$ satisfies (f1)-(f4). Let $\left(\lambda_{1}(s), u_{1}(s, x)\right), s \in$ $\left(\alpha, g^{-1}(m)\right)$, be the branch of the monotone decreasing solutions of (2.1), and let $\mu_{1}(s)$ be the principal eigenvalue of $u_{1}(s, x)$.

1. If $f$ also satisfies (f5a), then, as $s \rightarrow M=g^{-1}(m), \lambda_{1}(s) \rightarrow \infty$, and

$$
\left|\mu_{1}(s)\right|=O\left(\lambda_{1}(s) e^{-K \lambda_{1}(s)}\right) \rightarrow 0,
$$

we have

$$
\sup _{0 \leq x \leq a}\left|u_{1}(s, x)-U_{1}\left(\sqrt{\lambda_{1}(s)}\left(x-x_{s}^{\alpha}\right)\right)\right| \rightarrow 0,
$$

where $x_{s}^{\alpha}$ is the unique point in $(0, a)$ such that $u_{1}\left(s, x_{s}^{\alpha}\right)=\alpha, \lim _{s \rightarrow M} x_{s}^{\alpha}=$ $a / 2, K>0$ is a constant only depending on $\left|f^{\prime}(m)\right|$ and $\left|f^{\prime}(M)\right|$, and $U_{1}$ is the unique solution of

$$
\left\{\begin{array}{l}
U^{\prime \prime}+f(U)=0, \quad x \in(-\infty, \infty), \\
\lim _{x \rightarrow-\infty} U(x)=M, \lim _{x \rightarrow \infty} U(x)=m, U(0)=\alpha .
\end{array}\right.
$$


2. If $f$ also satisfies (f5b), then as $s \rightarrow g^{-1}(m)<M, \lambda_{1}(s) \rightarrow \infty$, and

$$
\left|\mu_{1}(s)\right|=O\left(\lambda_{1}(s)\right) \rightarrow-\infty,
$$

we have

$$
\sup _{0 \leq x \leq a}\left|u_{1}(s, x)-U_{2}\left(\sqrt{\lambda_{1}(s)} x\right)\right| \rightarrow 0,
$$

where $U_{2}$ is the unique solution of

$$
\left\{\begin{array}{l}
U^{\prime \prime}+f(U)=0, \quad x \in(-\infty, \infty), \\
\lim _{x \rightarrow \pm \infty} U(x)=m, U(0)=g^{-1}(m) .
\end{array}\right.
$$

For monotone increasing solutions on $\Sigma_{1}^{-}$, similar results hold with monotone increasing homoclinic and heteroclinic solutions.

Proof of Proposition 2.6. We only indicate where proofs can be found in previous works on slow-motion of the Allen-Cahn or Cahn-Hilliard equation and studies of the spike layer solutions. For balanced $f$, (2.22) can be obtained by a blow-up argument which will be used repeatedly in Section 6, and for this case, a proof can be found in $\mathrm{ABF}$, Appendix, pages 129-130. The estimate (2.21) is proved in ABF, Proposition A1 (pages 128-130) (see also [CGS], CP]). We should notice that the equation in $\mathrm{ABF}$ is slightly different from (2.1): instead of $u_{1}(s, \cdot)$, they consider $u^{\xi}$, which also satisfies an integral constraint. But the same proof can be carried over without essential change. For unbalanced $f,(2.25)$ can also be proved by a blow-up argument; see BFi], Lemma 6 (page 996), or [NT2], BDS for the higher-dimensional case. The proof of (2.24) can be found in BFi] Theorem 4 (pages 995-999), or [BDS], [BS] for the higher-dimensional case.

The last question we discuss in this section is the monotonicity of $\lambda_{1}(s)$. First we consider the direction of the bifurcation at $\left(\lambda_{1} / f^{\prime}(\alpha), \alpha\right)$. Here we derive some general formulas. Let $(\lambda(s), u(s)),|s| \leq \delta$, be a solution curve of (1.4) with a general domain $\Omega$ bifurcating from $\left(\lambda_{*}, \alpha\right)$, where $\lambda_{*} f^{\prime}(\alpha)=\lambda_{k}$, a simple eigenvalue of $-\Delta$ in $H^{1}(\Omega)$. Differentiating (1.4) with respect to $s$ twice, and evaluating at $s=0$, we get

$$
\Delta u_{s s}(0)+\lambda(0) f^{\prime}(\alpha) u_{s s}(0)+2 \lambda^{\prime}(0) f(\alpha) w+\lambda(0) f^{\prime \prime}(\alpha) w^{2}=0,
$$

$\partial_{n} u_{s s}(0)=0$ on $\partial \Omega$, where $w=u^{\prime}(0)$ is a solution of

$$
\Delta w+\lambda(0) f^{\prime}(\alpha) w=0, \partial_{n} w=0 \text { on } \partial \Omega .
$$

Using (2.27) and (2.28), we get

$$
\lambda^{\prime}(0)=-\frac{\lambda_{*} f^{\prime \prime}(\alpha) \int_{\Omega} w^{3} d x}{2 f^{\prime}(\alpha) \int_{\Omega} w^{2} d x}=-\frac{\lambda_{k} f^{\prime \prime}(\alpha) \int_{\Omega} w^{3} d x}{2\left[f^{\prime}(\alpha)\right]^{2} \int_{\Omega} w^{2} d x} .
$$

However, very often (always true for one dimension) $\int_{\Omega} w^{3} d x=0$; so it is necessary to compute $\lambda^{\prime \prime}(0)$. Here we assume $f \in C^{3}$ near $u=\alpha$. Then, differentiating (1.4) further, if $\lambda^{\prime}(0)=0$, then we get

$$
\begin{aligned}
\Delta u_{s s s}(0) & +\lambda_{*} f^{\prime}(\alpha) u_{s s s}(0)+3 \lambda^{\prime \prime}(0) f^{\prime}(\alpha) w \\
& +\lambda_{*} f^{\prime \prime \prime}(\alpha) w^{3}+3 \lambda_{*} f^{\prime \prime}(\alpha) w u_{s s}(0)=0,
\end{aligned}
$$


and $\partial_{n} u_{s s s}(0)=0$ on $\partial \Omega$, where $u_{s s}(0)$ satisfies (2.27) with $\lambda^{\prime}(0)=0$. Then

$$
\begin{aligned}
\lambda^{\prime \prime}(0) & =-\frac{\lambda_{*} f^{\prime \prime \prime}(\alpha) \int_{\Omega} w^{4} d x+\lambda_{*} f^{\prime \prime}(\alpha) \int_{\Omega} w^{2} u_{s s}(0) d x}{3 f^{\prime}(\alpha) \int_{\Omega} w^{2} d x} \\
& =-\frac{\lambda_{k} f^{\prime \prime \prime}(\alpha) \int_{\Omega} w^{4} d x+3 \lambda_{k} f^{\prime \prime}(\alpha) \int_{\Omega} w^{2} u_{s s}(0) d x}{3\left[f^{\prime}(\alpha)\right]^{2} \int_{\Omega} w^{2} d x} .
\end{aligned}
$$

Indeed, the formulas (2.29) and (2.31) hold for more general bifurcation problems; see Shi [S1], Section 4 . In the special case $\Omega=(0, a)$ we have $w(x)=\cos (k \pi x / a)$; so $\lambda_{k}^{\prime}(\alpha)=0$, and $u_{s s}(0)$ is the solution of

$$
v^{\prime \prime}+\left(\frac{k \pi}{a}\right)^{2} v+\frac{(k \pi)^{2} f^{\prime \prime}(\alpha)}{a^{2} f^{\prime}(\alpha)} \cos ^{2}\left(\frac{k \pi x}{a}\right)=0, v^{\prime}(0)=v^{\prime}(a)=0 .
$$

From simple calculations, we have $u_{s s}(0)=\left[f^{\prime}(\alpha)\right]^{-1} f^{\prime \prime}(\alpha)\left[\frac{1}{3} \cos ^{2}(k \pi x / a)-\frac{2}{3}\right]$. Then by computation, we get

$$
\lambda^{\prime \prime}(\alpha)=-\left(\frac{k \pi}{a}\right)^{2} \cdot \frac{3 f^{\prime}(\alpha) f^{\prime \prime \prime}(\alpha)-5\left[f^{\prime \prime}(\alpha)\right]^{2}}{12\left[f^{\prime}(\alpha)\right]^{3}} .
$$

Thus $\lambda^{\prime \prime}(\alpha)$ is determined by the value of $3 f^{\prime} f^{\prime \prime \prime}-5\left(f^{\prime \prime}\right)^{2}$ at $u=\alpha$. A similar formula was derived by Schaaf [Sc] (page 8) using the time-mapping formula and Korman Kor using a calculation similar to ours. For the monotonicity of $\lambda_{1}^{\prime}(s)$ at $s \neq \alpha$, we recall the results of [Sc] (page 50):

Theorem 2.7. Let $f$ satisfy $(f 1)-(f 5)$, and $f \in C^{3}(m, M)$. Let $\left(\lambda_{k}(s), u_{k}(s, x)\right)$ be as in Proposition 2.1

1. If

$$
(u-\alpha) \frac{d}{d u}\left[\frac{f(u)}{u-\alpha}\right]<(>) 0 \text { for } u \in(m, M) \backslash\{\alpha\},
$$

then $(s-\alpha) \lambda_{k}^{\prime}(s)>(<) 0$ for $s \in(m, M) \backslash\{\alpha\}$.

2. If, for $u \in(m, M)$,

$$
\begin{aligned}
& 3 f^{\prime}(u) f^{\prime \prime \prime}(u)-5\left[f^{\prime \prime}(u)\right]^{2}<0, \text { when } f^{\prime}(u)>0, \\
& f(u) f^{\prime \prime}(u)-3\left[f^{\prime}(u)\right]^{2} \leq 0, \text { when } f^{\prime}(u)<0, \\
& f^{\prime \prime}(u) \neq 0, \text { when } f^{\prime}(u)=0, \\
& \text { then }(s-\alpha) \lambda_{k}^{\prime}(s)>0 \text { for } s \in(m, M) \backslash\{\alpha\} \text {. }
\end{aligned}
$$

For the case of $f(u)=-(u-m)(u-M)(u-\alpha)$, it was shown by Smoller and Wasserman $[\mathrm{SmW}]$ that $\lambda_{1}^{\prime}(s)>0$ for $s \in S$, and we can also show that $f(u)$ satisfies (2.34); then the theorem above also applies here. For $f(u)=-u+u^{p}$, $u>0, p>1$, we have $g^{-1}(m)=\sqrt[p-1]{(p+1) / 2}$ and $\alpha=1$. It is easy to verify that $f(u)$ satisfies (2.35); thus $\lambda_{1}^{\prime}(s)>0$ for $s \in S=(1, \sqrt[p-1]{(p+1) / 2})$. Thus, for all examples in the introduction, $\Sigma_{1}$ is always a monotone increasing solution curve with Morse index 1 . Finally we remark that even when $f$ does not satisfy the conditions in Theorem [2.7] $\lambda_{k}^{\prime}(s)>0$ is still true when $s \rightarrow g^{-1}(m)$. In particular, as $\lambda \rightarrow \infty$, (2.1) has a unique monotone decreasing solution if $f$ satisfies (f1)-(f5). This fact will be used in Section 6 . 


\section{Primary Bifurcations from the Trivial Solutions}

From this section on, we consider

$$
\begin{cases}\Delta u+\lambda f(u)=0, & (x, y) \in \Omega \equiv(0, a) \times(0, b), \\ \frac{\partial u}{\partial n}=0, & (x, y) \in \partial \Omega,\end{cases}
$$

where $a, b>0$. We assume $f$ satisfies (f1)-(f4), and (f5a) or (f5b). In this section we discuss the solutions of (3.1) which bifurcate from the line of the trivial solutions $\Sigma_{0,0}=\{(\lambda, \alpha): \lambda>0\}$. The eigenvalue problem

$$
\begin{cases}\Delta \Psi+\eta f^{\prime}(\alpha) \Psi=0, & (x, y) \in \Omega \equiv(0, a) \times(0, b), \\ \frac{\partial \Psi}{\partial n}=0, & (x, y) \in \partial \Omega,\end{cases}
$$

has eigenpairs of the form $(k, l \in\{0\} \cup \mathbf{N})$

$$
\eta_{k, l}=\left(\frac{k^{2}}{a^{2}}+\frac{l^{2}}{b^{2}}\right) \frac{\pi^{2}}{f^{\prime}(\alpha)}, \quad \Psi_{k, l}(x, y)=\cos \left(\frac{k \pi x}{a}\right) \cos \left(\frac{l \pi y}{b}\right) .
$$

We notice that if $\Omega$ is a simple rectangle (meaning $a / b$ is irrational), then all $\eta_{k, l}$ are simple eigenvalues, but if $\Omega$ is not a simple rectangle, then $\eta_{k, l}$ may not be simple. We study the bifurcations of the solutions under periodicity. The periodicity of $\Psi_{k, l}$ can be characterized by

$$
\begin{aligned}
\Psi_{k, l}\left(x+\frac{2 a}{k}, y\right) & =\Psi_{k, l}(x, y), \\
\Psi_{k, l}\left(x, y+\frac{2 b}{l}\right) & =\Psi_{k, l}(x, y), \\
\Psi_{k, l}\left(\frac{a}{k}-x, \frac{b}{l}-y\right) & =\Psi_{k, l}(x, y),
\end{aligned}
$$

as long as $k \neq 0$ and $l \neq 0$ and these points are in $\Omega$. When $k=0$ or $l=0$, the periodicity becomes homogeneity:

$$
\begin{aligned}
& \Psi_{k, 0}\left(x, y_{1}\right)=\Psi_{k, 0}\left(x, y_{2}\right), \\
& \Psi_{0, k}\left(x_{1}, y\right)=\Psi_{0, k}\left(x_{2}, y\right),
\end{aligned}
$$

for any $x, x_{i} \in(0, a)$ and $y, y_{i} \in(0, b)$. A solution of (3.1) satisfies

$$
\frac{\partial u}{\partial n}=0 \quad \text { on } \quad \partial \Omega \text {. }
$$

Let $X=C^{2, \alpha}(\bar{\Omega})$ and $Y=C^{\alpha}(\bar{\Omega})$. We define, for $k \geq 1, l \geq 1$,

$$
\begin{aligned}
X^{k, 0} & =\{u \in X: u \text { satisfies (3.3), and (3.8) }\}, \\
X^{0, l} & =\{u \in X: u \text { satisfies (3.4), and (3.8) }\}, \\
X_{k, l} & =\{u \in X: u \text { satisfies (3.3), (3.4), (3.5) and (3.8) }\}, \\
X_{k, 0} & =\{u \in X: u \text { satisfies (3.3), (3.6) and (3.8) }\}, \\
X_{0, l} & =\{u \in X: u \text { satisfies (3.4), (3.6) and (3.8) }\} .
\end{aligned}
$$

Aso we define $Y_{k, l}$ and $Y^{k, l}$ by replacing $X$ by $Y$ in all definitions above. Since the periodicity and the homogeneity defined above are all linear properties, and $X_{k, l}$ is 
closed in $X$, then $X_{k, l}$ is a well-defined Banach space itself. We also observe that

$$
\begin{aligned}
& X_{k, 0} \subset X^{k, 0}, X_{0, l} \subset X^{0, l}, X^{k m, 0} \subset X^{k, 0}, \\
& X^{0, l m} \subset X^{0, l}, X_{k m, p} \subset X_{k, p}, X_{p, l m} \subset X_{p, l},
\end{aligned}
$$

for $k, l, m \geq 1, p \geq 0$. We define a map $F(\lambda, u)=\Delta u+\lambda f(u), u \in X_{k, l}$. Then $F(\lambda, u) \in Y_{k, l}$ if $u \in X_{k, l}$. The same is true for $X^{0, l}$ and $Y^{0, l}$, or $X^{k, 0}$ and $Y^{k, 0}$. We can also regard the solutions of (3.1) as the doubly periodic solutions of $\Delta u+\lambda f(u)=0$ in $\mathbf{R}^{2}$, since we can extend a solution $u$ of (3.1) evenly with respect to both axes, then extend it periodically to $\mathbf{R}^{2}$.

Proposition 3.1. Suppose that $f$ satisfies (f1) and $f^{\prime}(\alpha)>0$. For any $k, l \in$ $\mathbf{N} \cup\{0\}, k+l>0,\left(\eta_{k, l}, \alpha\right)$ is a bifurcation point for (3.1), and there is a continuum of nontrivial solutions $\Sigma_{k, l} \subset \mathbf{R}^{+} \times X_{k, l}$ bifurcating from $\left(\eta_{k, l}, \alpha\right)$. Near $\left(\eta_{k, l}, \alpha\right)$, $\Sigma_{k, l}=\left\{\left(\lambda_{k, l}(t), \alpha+t \Psi_{k, l}+o(|t|)\right): t \in(-\delta, \delta)\right\}$ with $\lambda_{k, l}(0)=\eta_{k, l}$. Moreover, either $\Sigma_{k, l}$ is unbounded in $\mathbf{R}^{+} \times X_{k, l}$, or $\Sigma_{k, l}$ meets $\mathbf{R}^{+} \times\{\alpha\}$ at $\eta \neq \eta_{k, l}$. The same results hold for $X^{k, 0}$ and $X^{0, l}$. We denote the continuum in $X^{k, 0}$ by $\Sigma^{k, 0}$, and the continuum in $X^{0, l}$ by $\Sigma^{0, l}$. Then $\Sigma^{k, 0} \supseteq \Sigma_{k, 0}$ and $\Sigma^{0, l} \supseteq \Sigma_{0, l}$.

Proof. Consider $F(\lambda, u)=\Delta u+\lambda f(u), u \in X_{k, l}$. Let $\left(\lambda_{0}, u_{0}\right)=\left(\eta_{k, l}, \alpha\right)$. Then $N\left(F_{u}\left(\lambda_{0}, u_{0}\right)\right)=\operatorname{span}\left\{\Psi_{k, l}\right\}$, which is one-dimensional, and $R\left(F_{u}\left(\lambda_{0}, u_{0}\right)\right)=\{v \in$ $\left.Y_{k, l}: \int_{\Omega} \Psi_{k, l} v d x d y=0\right\}$, which is codimension one. Finally, $F_{\lambda u}\left(\lambda_{0}, u_{0}\right) \Psi_{k, l}=$ $f^{\prime}(\alpha) \Psi_{k, l} \notin R\left(F_{u}\left(\lambda_{0}, u_{0}\right)\right)$, since $\int_{\Omega} f^{\prime}(\alpha) \Psi_{k, l}^{2} d x d y \neq 0$. Therefore the results follows from the global bifurcation result of $[\underline{\mathrm{R}}]$ and Theorem 1.7 of [CR]. The results for $X^{k, 0}$ and $X^{0, l}$ are proved in the same way, and (3.9) implies $\Sigma^{k, 0} \supseteq \Sigma_{k, 0}$ and $\Sigma^{0, l} \supseteq \Sigma_{0, l}$.

Remark 3.2.

1. The branch $\Sigma_{k, 0}$ consists of the semi-trivial solutions, which only depend on $x$. The solutions on $\Sigma^{k, 0}$ may also depend on $y$. In the next section, we will show that $\Sigma^{k, 0}$ is indeed a bigger set than $\Sigma_{k, 0}$ under certain conditions, and it will also include some secondary bifurcation sub-branches.

2. We define

$$
\begin{aligned}
& \Sigma_{k, l}^{+}=\left\{(\lambda, v) \in \Sigma_{k, l}: v(0,0)>\alpha\right\}, \\
& \Sigma_{k, l}^{-}=\left\{(\lambda, v) \in \Sigma_{k, l}: v(0,0)<\alpha\right\} .
\end{aligned}
$$

Clearly, $\Sigma_{k, 0}^{+}$is the branch of the semi-trivial solutions generated by the solutions on $\Sigma_{k}^{+}$of (1.2), and $\Sigma_{k, 0}^{-}$corresponds to $\Sigma_{k}^{-}$.

3. The symmetric group of the domain $\Omega$ is $G=\left\{1, R_{x}, R_{y}, R_{x} * R_{y}\right\}$ if $a \neq b$, where

$$
R_{x}(x, y)=(a-x, y), \quad R_{y}(x, y)=(x, b-y),
$$

and $*$ is the multiplication in the group. Clearly, if $u$ is a solution of (3.1), so is $g(u)$ for any $g \in G$. For all cases in Proposition 3.1 we have $G\left(\Sigma_{k, l}\right)=\Sigma_{k, l}$, since $\Sigma_{k, 0}$ and $\Sigma^{k, 0}$ are invariant under $R_{y}, \Sigma_{0, l}$ and $\Sigma^{0, l}$ are invariant under $R_{x}, \Sigma_{k, l}$ is invariant under $R_{x} * R_{y}$ for $k \geq 1, l \geq 1$ and $\Sigma_{k, l}^{+}=g\left(\Sigma_{k, l}^{-}\right)$for all cases where $g$ is the unique element in $G$ which has a nontrivial operation on the $\Sigma_{k, l}$. 
If $\eta_{k, l}$ are all simple eigenvalues, then Proposition 3.1 describes all possible primary bifurcations from the trivial solutions $\{(\lambda, \alpha)\}$. But if $a / b$ is a rational number, then it is possible that $\eta_{k, l}=\eta_{s, t}$ for some $k \neq s$ and $l \neq t$. The bifurcation at a nonsimple eigenvalue is more complicated. There are no complete results in this case, but partial results have been obtained by Kielhöfer [Ki] and Mei [Me]; see also [MM] for numerical results. In [S4], we study other primary bifurcations from the line of the trivial solutions when $\Omega=(0, a) \times(0, a)$, and their relations to the saddle solutions when $f$ is a balanced nonlinearity.

\section{Secondary Bifurcations from the Semi-Trivial Solutions}

Suppose that $f$ satisfies (f1)-(f5). From Proposition 3.1 for each $k>0$, there are two semi-trivial solution branches, $\Sigma_{k, 0}$ and $\Sigma_{0, k}$, bifurcating from $\eta_{k, 0}=\frac{k^{2} \pi^{2}}{a^{2} f^{\prime}(\alpha)}$ and $\eta_{0, k}=\frac{k^{2} \pi^{2}}{b^{2} f^{\prime}(\alpha)}$ respectively. On the other hand, there is a possibly larger solution continuum $\Sigma^{k, 0} \supset \Sigma_{k, 0}$. In this section, we study $\Sigma^{k, 0}$. For simplicity, we only consider the case $k=1$, and we also assume that if $\Sigma_{1}^{+}=\left\{\left(\lambda_{1}(s), u_{1}(s, x)\right)\right.$ : $s \in S\}$, then $\lambda_{1}^{\prime}(s)>0$ for $s \in S$. (Recall from the end of Section 2 that this is true for all examples introduced in Section 1.) From Lemma 2.3. $M\left(u_{1}(s, \cdot)\right)=1$ for all $s \in S$. Thus $\mu_{1}(s)<0$ and $\mu_{2}(s)>0$ for $s \in S$. Since $\lambda_{1}^{\prime}(s)>0$ for $s \in S=\left(\alpha, g^{-1}(m)\right)$, then $\lambda$ and $s$ can be viewed as two equivalent parameters of $\Sigma_{1,0}^{+}$. Thus a family of functions with parameter $s$ can also be regarded as a family of functions with parameter $\lambda$.

For $s \in(m, M)$, the semi-trivial solution on $\Sigma_{1,0}$ is of form $v_{1}(s, x, y)=u_{1}(s, x)$, and $\Sigma_{1,0}=\left\{\left(\lambda_{1}(s), v_{1}(s, x, y)\right): s \in S \cup g(S)\right\}$ is a solution curve of (3.1). Sometimes we also use $v_{1}(\lambda, x, y)$. We compute the Morse index of $v_{1}(s, x, y)$. The Morse index $M\left(v_{1}(s, x, y)\right)$ is the number of negative eigenvalues of

$$
\begin{cases}\Delta \phi+\lambda(s) f^{\prime}\left(v_{1}(s, x, y)\right) \phi=-\xi \phi, & (x, y) \in \Omega \\ \frac{\partial \phi}{\partial n}=0, & (x, y) \in \partial \Omega .\end{cases}
$$

To compute the eigenvalues of (4.1), we use the method of separation of variables. Let $\phi(x, y)=X(x) Y(y)$. Then $X$ and $Y$ satisfy

$$
\left\{\begin{array}{l}
X^{\prime \prime}+\lambda(s) f^{\prime}\left(u_{1}(s, x)\right) X=-K X, \quad X^{\prime}(0)=X^{\prime}(a)=0 \\
Y^{\prime \prime}+\xi Y=K Y, \quad Y^{\prime}(0)=Y^{\prime}(b)=0 .
\end{array}\right.
$$

Thus $K=\mu_{k}(s)$ and $\xi=K+\lambda_{i}=\mu_{k}(s)+\frac{i^{2} \pi^{2}}{b^{2}}$. Recall that $\lambda_{i}=\frac{i^{2} \pi^{2}}{b^{2}}(i \geq 0)$ are the eigenvalues of (2.16) with length of interval $b$. We denote the eigenvalues of (4.1) by $\xi_{k, i}(s)=\mu_{k}(s)+\frac{i^{2} \pi^{2}}{b^{2}}$, for $k \geq 1$ and $i \geq 0$, and the eigenfunction corresponding to $\xi_{k, i}(s)$ is $\phi_{k}(s, x) \cos (i \pi y / b)$, where $\phi_{k}(s, x)$ is the eigenfunction of $\mu_{k}(s)$. The principal eigenvalue $\xi_{1,0}(s)=\mu_{1}(s)$ is always negative, and since we assume that $\mu_{2}(s)>0$, then the only possible negative eigenvalues are $\xi_{1, i}(s)$ for $0 \leq i \leq R(s)$, where

$$
R(s)=\left[\frac{b \sqrt{-\mu_{1}(s)}}{\pi}\right]
$$


where $[t]=$ the greatest integer less than $t$, and clearly $M\left(v_{1}(s, x, y)\right)=R(s)+1$. (Note that $[n]=n-1$ if $n$ is an integer, since the Morse index only counts the strictly negative eigenvalues.) Now we identify the bifurcation points on the branch $\Sigma_{1,0}$.

Lemma 4.1. Suppose that $f$ satisfies (f1)-(f4).

1. If $f$ also satisfies (f5a), then there exist an integer $N>0$ and $2 N$ numbers $\Lambda_{k}^{ \pm}, k=1,2, \cdots, N$, such that

$$
\frac{\pi^{2}}{a^{2} f^{\prime}(\alpha)}<\Lambda_{1}^{+}<\Lambda_{2}^{+}<\cdots<\Lambda_{N}^{+} \leq \Lambda_{N}^{-}<\cdots<\Lambda_{2}^{-}<\Lambda_{1}^{-}<\infty .
$$

When $\lambda_{1}(s)=\Lambda_{k}^{ \pm}, M\left(v_{1}(s, x, y)\right)=k$ and 0 is the $(k+1)$-th eigenvalue.

2. If $f$ also satisfies (f5b), then there exists a sequence $\Lambda_{k}, k \in \mathbf{N}$, such that

$$
\frac{\pi^{2}}{a^{2} f^{\prime}(\alpha)}<\Lambda_{1}<\Lambda_{2}<\cdots<\Lambda_{N}<\cdots<\infty .
$$

When $\lambda_{1}(s)=\Lambda_{k}, M\left(v_{1}(s, x, y)\right)=k$ and 0 is the $(k+1)$-th eigenvalue.

Proof. Consider the function $\mu_{1}(s): S \equiv\left(\alpha, g^{-1}(m)\right) \rightarrow(-\infty, 0)$. Then

$$
\lim _{s \rightarrow \alpha^{+}} \mu_{1}(s)=0 \text {, }
$$

and from Proposition 2.6

$$
\lim _{s \rightarrow g^{-1}(m)^{-}} \mu_{1}(s)= \begin{cases}0 & \text { if }(\mathrm{f} 5 \mathrm{a}) \text { is satisfied, } \\ -\infty & \text { if (f5b) is satisfied. }\end{cases}
$$

In the former case, assume that $M_{1}=\max _{s \in S_{+}} \sqrt{-\mu_{1}(s)}$ and $N=\left[b M_{1} / \pi\right]$; then there exists $s_{k}^{ \pm} \in S, k=1,2, \cdots, N$, such that $\mu_{1}\left(s_{k}^{ \pm}\right)=-k^{2} \pi^{2} / b^{2}, \mu_{1}^{\prime}\left(s_{k}^{+}\right) \geq 0$ and $\mu_{1}^{\prime}\left(s_{k}^{-}\right) \leq 0$. Then $\Lambda_{k}^{ \pm}=\lambda_{1}\left(s_{k}^{ \pm}\right)$satisfies the desired properties. The other case is similar.

Now we are ready to prove the result on the local secondary bifurcations along $\Sigma_{1,0}$ at $\lambda=\Lambda_{k}^{ \pm}$or $\Lambda_{k}$.

Proposition 4.2. Suppose that $f$ satisfies $(f 1)-\left(f_{4}\right)$.

1. If (f5b) is satisfied, $\lambda_{1}\left(s_{k}\right)=\Lambda_{k}$, and $\mu_{1}^{\prime}\left(s_{k}\right) \neq 0$, then a continuum of solutions of (3.1) bifurcates from $\Sigma_{1,0}^{+}$at $\left(\lambda_{0}, u_{0}\right)=\left(\Lambda_{k}, v_{1}\left(s_{k}\right)\right)$, and the solution set of (3.1) near $\left(\lambda_{0}, u_{0}\right)$ consists of two parts: the semi-trivial branch $\left\{\left(\lambda_{1}(s), v_{1}(s)\right)\right\}$ and $\Sigma_{1,0, k}^{+}=\left\{\left(\lambda(t), v_{1}(\lambda(t))+t \Phi\left(s_{k}\right)+o(|t|)\right)\right\},|t| \leq \delta, \lambda(0)=$ $\Lambda_{k}, \Phi(s, x, y)=\phi_{1}(s, x) \cos (k \pi y / b)$; for any $(\lambda, u) \in \Sigma_{1,0, k}^{+}, u-v_{1}(\lambda) \in X^{0, k}$, either $\left\{\left(\lambda, u-v_{1}(\lambda)\right):(\lambda, u) \in \Sigma_{1,0, k}^{+}\right\}$is unbounded, or $\Sigma_{1,0, k}^{+}$meets $\Sigma_{1,0}^{+}$at another point $\lambda=\Lambda_{j}$ (see Fig. 5).

2. If (f5a) is satisfied, the same result holds near $\Lambda_{k}^{ \pm}$(see Fig. 4).

Proof. Since $\mu_{2}(s)>0$, then the $(k+1)$-th eigenvalue of $v_{1}(s)$ must be $\xi_{1, k}=$ $\mu_{1}(s)+\frac{k^{2} \pi^{2}}{b^{2}}=0$ with eigenfunction $\Phi(s, x, y)=\phi_{1}(s, x) \cos \left(\frac{k \pi y}{b}\right)$. Define an operator $F(\lambda, u):\left(\Lambda_{k}-\delta, \Lambda_{k}+\delta\right) \times X^{0, k} \rightarrow Y^{0, k}$,

$$
F(\lambda, u)=\Delta u+\lambda f\left(u+v_{1}(\lambda)\right)-\lambda f\left(v_{1}(\lambda)\right),
$$


where $\delta>0$ is a small constant. The derivatives of $F$ are

$$
\begin{aligned}
& F_{u}(\lambda, u) w=\Delta w+\lambda f^{\prime}\left(u+v_{1}(\lambda)\right) w \\
& F_{\lambda}(\lambda, u)=f\left(u+v_{1}(\lambda)\right)-f\left(v_{1}(\lambda)\right)+\lambda f^{\prime}\left(u+v_{1}(\lambda)\right) \frac{\partial v_{1}(\lambda)}{\partial \lambda} \\
& \quad-\lambda f^{\prime}\left(v_{1}(\lambda)\right) \frac{\partial v_{1}(\lambda)}{\partial \lambda}, \\
& \quad F_{\lambda u}(\lambda, u) w=f^{\prime}\left(u+v_{1}(\lambda)\right) w+\lambda f^{\prime}\left(u+v_{1}(\lambda)\right) \frac{\partial v_{1}(\lambda)}{\partial \lambda} w .
\end{aligned}
$$

First we have $F(\lambda, 0) \equiv 0$ for $\lambda \in\left(\Lambda_{k}-\delta, \Lambda_{k}+\delta\right) ; N\left(F_{u}\left(\Lambda_{k}, 0\right)\right)=\operatorname{span}\{\Phi\}$, $\Phi=\Phi\left(s_{k}, x, y\right)=\phi_{1}\left(s_{k}, x\right) \cos (k \pi y / b) \in X^{0, k}$, which is one-dimensional; $F_{u}\left(\Lambda_{k}, 0\right)$ is a Fredholm operator with index 0 , so $\operatorname{codim} R\left(F_{u}\left(\Lambda_{k}, 0\right)\right)=\operatorname{dim} N\left(F_{u}\left(\Lambda_{k}, 0\right)\right)$ $=1$. From the Fredholm alternative, $v \in R\left(F_{u}\left(\Lambda_{k}, 0\right)\right)$ if and only if $\int_{\Omega} v \Phi d x d y=0$. Finally, $F_{\lambda u}\left(\Lambda_{k}, 0\right) \Phi \notin R\left(F_{u}\left(\Lambda_{k}, 0\right)\right)$ is equivalent to

$$
\int_{\Omega}\left[f^{\prime}\left(v_{1}(\lambda)\right) \Phi^{2}+\lambda f^{\prime}\left(v_{1}(\lambda)\right) \frac{\partial v_{1}(\lambda)}{\partial \lambda} \Phi^{2}\right] d x d y \neq 0 .
$$

Let $\left(\mu_{1}(\lambda), \phi_{1}(\lambda)\right)$ be the principal eigenpair of $u_{1}(\lambda, x)$. Then

$$
\begin{aligned}
& \int_{\Omega}\left[f^{\prime}\left(v_{1}(\lambda)\right) \Phi^{2}+\lambda f^{\prime}\left(v_{1}(\lambda)\right) \frac{\partial v_{1}(\lambda)}{\partial \lambda} \Phi^{2}\right] d x d y \\
= & \int_{0}^{a}\left[f^{\prime}\left(u_{1}\right) \phi_{1}^{2}(x)+\lambda f^{\prime}\left(u_{1}\right) \frac{\partial u_{1}}{\partial \lambda} \phi_{1}^{2}(x)\right] d x \cdot \int_{0}^{b}\left[\cos \left(\frac{k \pi y}{b}\right)\right]^{2} d y .
\end{aligned}
$$

Also, $\left(\mu_{1}(\lambda), \phi_{1}(\lambda)\right)$ satisfies

$$
\left\{\begin{array}{l}
\phi_{1}^{\prime \prime}(\lambda)+\lambda f^{\prime}\left(u_{1}\right) \phi_{1}(\lambda)=-\mu_{1}(\lambda) \phi_{1}(\lambda), \quad x \in(0, a), \\
\phi_{1}^{\prime}(\lambda, 0)=\phi_{1}^{\prime}(\lambda, a)=0 .
\end{array}\right.
$$

Differentiating (4.9) with respect to $\lambda$, we have

$$
\left\{\begin{array}{c}
\left(\phi_{1}\right)_{\lambda}^{\prime \prime}+\lambda f^{\prime}\left(u_{1}\right)\left(\phi_{1}\right)_{\lambda}+f^{\prime}\left(u_{1}(\lambda)\right) \phi_{1}+\lambda f^{\prime \prime}\left(u_{1}\right)\left(u_{1}\right)_{\lambda} \phi_{1} \\
=-\mu_{1}\left(\phi_{1}\right)_{\lambda}-\left(\mu_{1}\right)_{\lambda} \phi_{1} \\
\left(\phi_{1}\right)_{\lambda}^{\prime}(\lambda, 0)=\left(\phi_{1}\right)_{\lambda}^{\prime}(\lambda, a)=0 .
\end{array}\right.
$$

Multiplying (4.9) by $\left(\phi_{1}\right)_{\lambda}$, (4.10) by $\phi_{1}$, subtracting and integrating over $(0, a)$, we obtain

$$
\begin{aligned}
& \int_{0}^{a}\left[f^{\prime}\left(u_{1}(\lambda, x)\right) \phi_{1}^{2}(x)+\lambda f^{\prime}\left(u_{1}(\lambda, x)\right) \frac{\partial u_{1}(\lambda, x)}{\partial \lambda} \phi_{1}^{2}(x)\right] d x \\
= & -\frac{\partial \mu_{1}(\lambda)}{\partial \lambda} \int_{0}^{a} \phi_{1}^{2}(x) d x .
\end{aligned}
$$

Thus (4.7) is fulfilled if $\left(\mu_{1}\right)_{s}(s) \neq 0$ at $s=s_{k}$, since

$$
\frac{\partial \mu_{1}(s)}{\partial s}=\frac{\partial \mu_{1}(\lambda)}{\partial \lambda} \lambda^{\prime}(s)
$$

Therefore we can apply Theorem 1.7 of $[\mathrm{CR}$ to conclude that the solution set of (3.1) near $(\lambda, u)=\left(\Lambda_{k}, v_{1}\left(\Lambda_{k}, x, y\right)\right)$ consists of two parts: the semi-trivial branch $\left\{\left(\lambda(s), v_{1}(s, x, y)\right)\right\}$ and another branch

$$
\Sigma_{1,0, k}^{+}=\{(\lambda(t), u(\lambda(t), x, y))\}=\left\{\left(\lambda(t), v_{1}(\lambda(t), x, y)+t \Phi\left(s_{k}, x, y\right)+o(|t|)\right)\right\},
$$


with $\Phi(s)=\phi_{1}(s, x) \cos (k \pi y / b)$. From the result of [ $[\mathrm{R}], \Sigma_{1,0, k}^{+}$is a global continuum, and $\left.v(t, x, y)=u(\lambda(t), x, y)-v_{1}(\lambda(t), x, y)\right) \in X^{0, k} ;\{(\lambda(t), v(t))\}$ is either unbounded in $\mathbf{R}^{+} \times X^{0, k}$, or $\{(\lambda(t), u(t))\}$ meets another point on $\Sigma_{1,0}^{+}$. The result for (f5a) is similar.

\section{Remark 4.3.}

1. For unbalanced $f$, there is always an infinite sequence of bifurcation points for any rectangle $(0, a) \times(0, b)$. But for the balanced case, we only know that the "mushrooms" exist when $b / a$ is sufficiently large from (4.3), and there are more $y$-direction secondary bifurcation branches when $b / a$ is larger. It is not clear if the secondary bifurcations occur when $a=b$.

2 . Given $a>0$, the condition $\mu_{1}^{\prime}(s) \neq 0$ at the bifurcation point (where $\mu_{1}(s)=$ $\left.k^{2} \pi^{2} / b^{2}\right)$ is true for all $b>0$ except maybe a set of zero measure, by Sard's Theorem. Therefore, the bifurcations in Proposition 4.2 occur for generic rectangles. Moreover, $\mu_{1}^{\prime}(s) \neq 0$ can be weakened to $\mu_{1}(s)+k^{2} \pi^{2} / b^{2}<0$ for $s \in\left(s_{0}-\delta, s_{0}\right)$, and $\mu_{1}(s)+k^{2} \pi^{2} / b^{2}>0$ for $s \in\left(s_{0}, s_{0}+\delta\right)$, or vice versa. We can apply bifurcation theory based on the Leray-Schauder degree theory instead of Theorem 1.7 of [CR (which is based on the implicit function theorem) to prove that a bifurcation occurs there.

3. The assumption $\lambda_{1}^{\prime}(s)>0$ for all $s>\alpha$ is not restrictive. If $\lambda_{1}(s)$ is not monotone, there are more bifurcation points. Also $\lambda_{1}^{\prime}(s)>0$ as $s \rightarrow g^{-1}(m)^{-}$, so there are always infinitely many bifurcation points for the unbalanced case.

4. If $\mu_{1}^{\prime}(s)<0$ for all $s \in\left(\alpha, g^{-1}(m)\right)$ in the unbalanced case, then $\Lambda_{k}$ is unique for each $k$. If $\mu_{1}(s)$ is not monotone, then there are more bifurcation points. Similarly, for the balanced case, $\Lambda_{k}^{ \pm}$is unique if $\mu_{1}(s)$ has only one critical point.

5. It is easy to do a similar analysis on the secondary bifurcations from $\Sigma_{0, k}$ and $\Sigma_{k, 0}$ for $k>1$, see Remark 2.4 But they are all symmetric extensions of solutions on $\Sigma_{1,0, n}$.

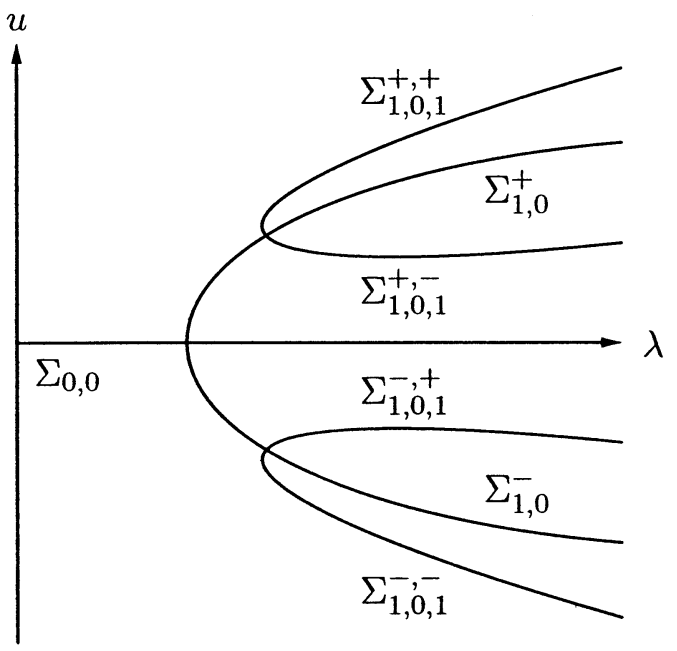

FIGURE 9. Labelling of the solution branches for unbalanced $f$ 
6. We can further decompose $\Sigma_{1,0, k}^{+}$:

$$
\Sigma_{1,0, k}^{+}=\Sigma_{1,0, k}^{+,+} \cup \Sigma_{1,0, k}^{+,-},
$$

where $\Sigma_{1,0, k}^{+,+}$is the subcontinuum such that $t>0$ and $\Sigma_{1,0, k}^{+,-}$with $t<0$ in Proposition 4.2 (See Fig. 9 for an illustration of the labelling of the solution branches.) For $f$ satisfying (f5a), we use the notation $\Sigma_{1,0, k+}^{+,+}$for the branch with $t>0$ bifurcating from $\Lambda_{k}^{+}$on $\Sigma_{1,0}^{+}$.

\section{Global Properties of the Solution Branches}

We have shown that there are two levels of bifurcations for (3.1). First, from $\Sigma_{0,0}=\{(\lambda, \alpha)\}$, there is a continuum $\Sigma_{k, l} \in \mathbf{R}^{+} \times X_{k, l}$ bifurcating at $\lambda=\eta_{k, l}$. Second, at least on $\Sigma_{0, k}$ or $\Sigma_{k, 0}$, there are continua $\Sigma_{0, k, l}$ or $\Sigma_{k, 0, l}$ bifurcating out.

Theorem 5.1. Suppose that $\Sigma_{k, l}, \Sigma_{0, k, l}$ and $\Sigma_{k, 0, l}$ are the solution branches obtained in Sections 3 and 4. We assume that $\Lambda_{k}^{ \pm}$(balanced case) and $\Lambda_{k}$ (unbalanced case) are unique.

1. If $f$ satisfies (f5a) and $b \neq l a$, then

$$
\Sigma_{1,0, l+}^{+}=\Sigma_{1,0, l-}^{+} \text {, which we call } \Sigma_{1,0, l}^{+} \text {from now on, }
$$

and for $l_{1} \neq l_{2}, \Sigma_{1,0, l_{1} \pm}^{ \pm} \cap \Sigma_{1,0, l_{2} \pm}^{ \pm}=\emptyset ; \Sigma_{1,0, l}^{+}$is bounded, and $\Sigma_{1,0, l}^{+} \cap \Sigma_{1,0}^{+}$are exactly the two bifurcation points. (See Figure 4.)

2. If $f$ satisfies (f5b), then for $l_{1} \neq l_{2}, \Sigma_{1,0, l_{1}}^{ \pm} \cap \Sigma_{1,0, l_{2}}^{ \pm}=\emptyset ; \Sigma_{1,0, l}$ is unbounded and $\Sigma_{1,0, l}^{ \pm} \cap \Sigma_{1,0}^{+}$is exactly the one bifurcation point. (See Figure 5.)

The theorem above shows that the secondary branches are bounded in the balanced case, connecting the branch from $\Lambda_{k}^{+}$and the branch from $\Lambda_{k}^{-}$. And we also prove here that all the secondary branches with different periods in the $y$ direction are separated, but it is still possible that some secondary branches are connected to other primary branches. Some separation results were also proved in Kielhöfer [Ki] for the similar Cahn-Hilliard equation, and it is not known if all primary branches are separated. Here we assume the uniqueness of $\Lambda_{k}$ or $\Lambda_{k}^{ \pm}$only for an easier statement. When $\Lambda_{k}^{ \pm}$is not unique in the balanced case, our proof of the theorem shows that all secondary branches are still bounded. When $\Lambda_{k}$ is not unique in the unbalanced case, we notice that generally for each $k \in \mathbf{N}$, the number of solution branches of the form $v_{1}(s)+X^{0, k}$ is an odd number, since the number of solutions of $\mu_{1}(s)=-k^{2} \pi^{2} / b^{2}$ is odd. Thus there is at least one unbounded branch with such mode.

The following lemma plays a key role in proving the theorem (results of this type were used in many works by Healey and Kielhöfer; see [HK], [Ki] and references therein).

Lemma 5.2. If $(\lambda, u)$ is a solution of (3.1) on $\Sigma_{1,0,1}^{ \pm, \pm}$(or $\Sigma_{1,0,1 \pm}^{ \pm, \pm}$), then $u_{x} \neq 0$ for $0<x<a, 0 \leq y \leq b$, and $u_{y} \neq 0$ for $0 \leq x \leq a, 0<y<b$.

Proof. Without loss of generality, we consider the solutions on $\Sigma_{1,0,1}^{+,+}$, and prove that $u_{x}<0$. We extend $u$ to the infinite strip $S_{a}=\{0<x<a,-\infty<y<\infty\}$ by even reflection. Then $u_{x}$ satisfies

$$
\Delta u_{x}+\lambda f^{\prime}(u) u_{x}=0, \quad \mathbf{x} \in S_{a}, u_{x}=0, \quad \mathbf{x} \in \partial S_{a} .
$$


We prove $u_{x}<0$ for $\mathbf{x} \in S_{a}$. Near the bifurcation point $\left(\Lambda_{1}, v_{1}\left(\Lambda_{1}\right)\right)$ we have $(\lambda, u)=\left(\lambda(t), v_{1}(\lambda(t))+t \Phi\left(s_{1}\right)+o(|t|)\right)$ with $t \in(0, \delta)$. Since $\left(\Phi\left(s_{1}\right)\right)_{x}<0$, $\left(\Phi\left(s_{1}\right)\right)_{y}<0$ and $\left(v_{1}\right)_{x}<0$ for $(x, y) \in \Omega$, then the conclusion holds for $(\lambda, u)$ near the bifurcation point. We also notice that for any direction $s$ entering $S_{a}$ transversally along $\partial S_{a}$, we have

$$
\frac{\partial u_{x}}{\partial s}(\mathbf{x})<0, \quad \mathbf{x} \in \partial S_{a}
$$

again when $(\lambda, u)$ is near the bifurcation point.

Suppose that the conclusion of the lemma is not true for some $(\lambda, u) \in \Sigma_{1,0,1}^{+,+}$; then by the connectedness of $\Sigma_{1,0,1}^{+,+}$there exists $\left(\lambda^{*}, u^{*}\right) \in \Sigma_{1,0,1}^{+,+}$such that $u_{x}^{*}(\mathbf{x}) \leq 0$ for all $\mathbf{x} \in \overline{S_{a}}$, and either

(a) there exists $\mathbf{x}_{0} \in S_{a}$ such that $u_{x}^{*}\left(\mathbf{x}_{0}\right)=0$, or

(b) there exists $\mathbf{x}_{0} \in \partial S_{a}$ such that $\left(\partial u_{x}^{*} / \partial s\right)\left(\mathbf{x}_{0}\right)=0$ for some $s$ transversal to $\partial S_{a}$.

However, from the maximum principle and the Hopf boundary lemma, neither can happen. Thus $u_{x}<0$ for any $(\lambda, u) \in \Sigma_{1,0,1}^{+,+}$. Similarly, $u_{y}<0$.

Proof of Theorem 5.1. First we consider the balanced case. From Lemma 5.2, we observe that if $(\lambda, u) \in \Sigma_{1,0, l \pm}^{ \pm}$, then the nodal set of $u_{x}$ and $u_{y}$ can be completely characterized by

$$
\begin{aligned}
& u_{x}(x, y)=0 \text { if and only if } x=0, a, y \in[0, b], \\
& u_{y}(x, y)=0 \text { if and only if } x \in[0, a], y=i b / l, 0 \leq i \leq l .
\end{aligned}
$$

Therefore if $l_{1} \neq l_{2}, \Sigma_{1,0, l_{1} \pm}^{ \pm} \cap \Sigma_{1,0, l_{2} \pm}^{ \pm}=\emptyset$. For fixed $l, \Sigma_{1,0, l+}^{+}$either is unbounded in its $X^{0, l}$ component (recall the form $v_{1}+X^{0, l}$ ) or meets another point on $\Sigma_{1,0}^{+}$from Rabinowitz's alternative $[\mathrm{R}$. Suppose that the former occurs; then we claim that $\Sigma_{1,0, l+}^{+}$is also unbounded in $\mathbf{R} \times X$. Define $\Sigma^{\prime}=\left\{(\lambda, w): w=u-v_{1}(\lambda),(\lambda, u) \in\right.$ $\left.\Sigma_{1,0, l+}^{+}\right\}$. If $\operatorname{proj}_{\lambda} \Sigma^{\prime}$ is unbounded, then $\operatorname{proj}_{\lambda} \Sigma_{1,0, l+}^{+}$is unbounded. If $\operatorname{proj}_{\lambda} \Sigma^{\prime}$ is bounded, then $\operatorname{proj}_{w} \Sigma^{\prime}$ is unbounded in $X$ and $\left\{v_{1}(\lambda): \lambda \in \operatorname{proj}_{\lambda} \Sigma^{\prime}\right\}$ is bounded in $X$; thus $\operatorname{proj}_{u} \Sigma_{1,0, l+}^{+}$is unbounded in $X$. Thus $\Sigma_{1,0, l+}^{+}$is unbounded in $\mathbf{R} \times X$. On the other hand, for any compact subset $K$ of $\mathbf{R}, \Sigma_{1,0, l+}^{+} \cap(K \times X)$ is also compact because of a priori estimates for the solutions of (3.1) with the balanced $f$. Therefore $\operatorname{proj}_{\lambda} \Sigma_{1,0, l+}^{+} \subset\left(\Lambda_{k}^{+}, \infty\right)$. However, from Theorem 6.6, (3.1) has no solution in the form $v_{1}(\lambda)+X^{0, l}$ except $v_{1}(\lambda)$ itself when $b \neq l a$, which is a contradiction, and thus $\Sigma_{1,0, l+}^{+}$is bounded. So $\Sigma_{1,0, l+}^{+}$must meet another point on $\Sigma_{1,0}^{+}$, which must be a bifurcation point with $\lambda=\Lambda_{j}^{ \pm}$. Since $\Sigma_{1,0, l_{1} \pm}^{ \pm} \cap \Sigma_{1,0, l_{2} \pm}^{ \pm}=\emptyset$ for $l_{1} \neq l_{2}$, then the other point has to be $\lambda=\Lambda_{k}^{-}$. The proof for the unbalanced case is similar: the key point is that each branch has different nodal structure, so they must all be unbounded.

\section{Remark 5.3.}

1. For the unbalanced case, if $f$ satisfies some extra conditions, then by the results of Ni and Takagi [NT1, all solutions of (3.1) are a priori bounded in $X$, and then the secondary bifurcation branches are bounded in the $u$ direction and unbounded in the $\lambda$ direction. We will prove in S5] that solutions on the secondary bifurcation branches are indeed spike layer solutions when $\lambda \rightarrow \infty$. 
2. We believe that the conclusions in Theorem 5.1 for balanced $f$ are still true when $b=k l$. For simplicity, we consider the case $b=a$. We only need to exclude the possibility of the solutions on $\Sigma_{1,0, l+}^{+}$being solutions with diagonal nodal lines. In [S4, we prove the existence of solutions with diagonal nodal lines in $\Omega=(0, a) \times(0, a)$, and these solutions are symmetric with respect to $x=y$. Thus, if we can show the uniqueness of such solutions, then $\Sigma_{1,0, l+}^{+}$must be bounded, since the solutions on $\Sigma_{1,0, l+}^{+}$are not symmetric with respect to $x=y$. But the uniqueness of such a solution is not yet known.

\section{Asymptotic Behaviors: The Balanced Case}

In this section, we consider the asymptotic behavior of monotone decreasing solutions as $\lambda \rightarrow \infty$ when $f$ is balanced. A monotone decreasing solution satisfies $u_{x} \leq 0$ and $u_{y} \leq 0$ for $(x, y) \in \Omega$. For convenience, we always use the conversion of parameters $\varepsilon=\lambda^{-1 / 2}$.

Define the nodal set of a solution $u$ to be

$$
\mathcal{N}(u)=\{\mathbf{x} \in \bar{\Omega}: u(\mathbf{x})=\alpha\} .
$$

For any solution $u$ of (3.1), $\mathcal{N}(u)$ is not an empty set since $\int_{\Omega} f(u) d x d y=0$. For a solution $u \in \Sigma_{1,0,1}^{+,+}, \mathcal{N}(u)$ is a simple monotone curve since $u_{x}<0$ and $u_{y}<0$. The nodal set $\mathcal{N}(u)$ separates $\Omega$ into two parts:

$$
\Omega^{+}(u)=\{\mathbf{x} \in \Omega: u(\mathbf{x})>\alpha\}, \quad \Omega^{-}(u)=\{\mathbf{x} \in \Omega: u(\mathbf{x})<\alpha\} .
$$

We first introduce some basic estimates for the solution $u$. The following result is well-known and can be proved by the sweeping principle. (See, for example, [D1].)

Lemma 6.1. Suppose that $f$ satisfies (f1)-( $f 4)$ and (f5a). For any $\tau \in(\alpha, M)$, there exist constants $K_{\tau}>0$ and $\lambda_{\tau}>0$ such that for any solution $(\lambda, u)$ of (3.1) with $\lambda>\lambda_{\tau}$, if $\mathbf{x} \in \Omega^{+}(u)$ and

$$
\operatorname{dist}(\mathbf{x}, \mathcal{N}(u)) \geq K_{\tau} \lambda^{-1 / 2}=K_{\tau} \varepsilon
$$

then $u(\mathbf{x}) \geq \tau$. The similar result holds for $\tau \in(m, \alpha)$ with $u(\mathbf{x}) \leq \tau$.

Proof. For $\tau \in(\alpha, M)$, we choose $A>0$ such that $f(u) \geq A(u-\alpha)$ for $u \in[\alpha, \tau]$. Define $d(\mathbf{x})=\operatorname{dist}(\mathbf{x}, \mathcal{N}(u)) \equiv \inf \{\operatorname{dist}(\mathbf{x}, \mathbf{y}): \mathbf{y} \in \mathcal{N}(u)\}$, and for $\mathbf{x}_{0} \in \Omega$ define a family of functions $(0 \leq t \leq A)$

$$
w_{t}(\mathbf{x})=\left\{\begin{array}{l}
t \phi_{1}\left(\frac{2\left(\mathbf{x}-\mathbf{x}_{0}\right)}{d\left(\mathbf{x}_{0}\right)}\right), \\
0, \quad \text { otherwise, }
\end{array} \quad \text { if } \operatorname{dist}\left(\mathbf{x}, \mathbf{x}_{0}\right) \leq \frac{1}{2} d\left(\mathbf{x}_{0}\right),\right.
$$

where $\phi_{1}$ is the positive principal eigenfunction of $-\Delta$ on $H_{0}^{1}\left(B^{2}\right)$ such that $\left\|\phi_{1}\right\|_{\infty}$ $=1$ and $B^{2}$ is the unit ball in $\mathbf{R}^{2}$. Then $w_{t}(\mathbf{x})$ is a subsolution of (3.1) if $\lambda>$ $4 \mu_{1} A^{-1} d\left(\mathbf{x}_{0}\right)^{-2}$, where $\mu_{1}$ is the principal eigenvalue of $-\Delta$ on $H_{0}^{1}\left(B^{2}\right)$. Therefore, by the sweeping principle, $u\left(\mathbf{x}_{0}\right) \geq \tau$ if $d\left(\mathbf{x}_{0}\right) \geq 2 \sqrt{\mu_{1} A^{-1}} \varepsilon$.

Another type of estimate is the exponential decay of the solution away from the nodal set.

Lemma 6.2. Suppose that $f$ satisfies $(f 1)-\left(f_{4}\right)$ and $(f 5 a)$. For any solution $(\lambda, u)$ of (3.1), if $\mathbf{x} \in \Omega^{+}(u)$, then

$$
|M-u(\mathbf{x})| \leq 2|M-\alpha| e^{-C_{1} d(\mathbf{x}) / \varepsilon},
$$


for some $C_{1}>0$, and $d(\mathbf{x})=\operatorname{dist}(\mathbf{x}, \mathcal{N}(u))$. Similarly, if $\mathbf{x} \in \Omega^{-}(u)$, then

$$
|m-u(\mathbf{x})| \leq 2|m-\alpha| e^{-C_{1} d(\mathbf{x}) / \varepsilon}
$$

Proof. We choose $\delta>0$ such that $f^{\prime}(u)<0$ for $u \in(m, m+\delta) \cup(M-\delta, M)$. Then from Lemma 6.1, there exists $R_{\delta}>0$ such that

$$
u(\mathbf{x}) \geq M-\delta \quad \text { if } \mathbf{x} \in \Omega^{+}, \text {and } d(\mathbf{x}) \geq R_{\delta} \varepsilon .
$$

We extend $u$ to the infinite strip $S=\{(x, y):|x| \leq a, y \in \mathbf{R}\}$ evenly. We define $\Omega_{\delta}^{+}=\left\{\mathbf{x} \in \Omega^{+}: d(\mathbf{x})>R_{\delta} \varepsilon\right\}$, and $\widetilde{\Omega_{\delta}^{+}}$to be the even reflection of $\Omega_{\delta}^{+}$to $S$. Then $u$ is a solution of (3.1) on $S$. For $\mathbf{x} \in \widetilde{\Omega_{\delta}^{+}}, u$ satisfies the equation

$$
\varepsilon_{j}^{2} \Delta(M-u)+\frac{f(M)-f(u)}{M-u}(M-u)=0 .
$$

From the mean-value theorem, we have

$$
\frac{f(M)-f(u(\mathbf{x}))}{M-u(\mathbf{x})}=f^{\prime}(\theta(\mathbf{x})) \leq-C<0, \quad \text { for } \mathbf{x} \in \widetilde{\Omega_{\delta}^{+}},
$$

where $u(\mathbf{x}) \leq \theta(\mathbf{x}) \leq M$. Then, by the well-known decay estimates for elliptic equations (see for example [NT2], page 840, Lemma 4.3),

$$
|M-u(\mathbf{x})| \leq 2\left(\max _{\mathbf{z} \in \widetilde{\Omega_{\delta}^{+}}}|M-u(\mathbf{z})|\right) e^{-C_{2} \varepsilon^{-1} d_{1}(\mathbf{x})}, \quad \mathbf{x} \in \widetilde{\Omega_{\delta}^{+}},
$$

where $d_{1}(\mathbf{x})$ is the distance from $\mathbf{x}$ to the boundary of $\widetilde{\Omega_{\delta}^{+}}$and $C_{2}>0$ is independent of $\varepsilon, u$ and $\mathbf{x}$. For $\mathbf{x} \in \Omega_{\delta}^{+}$, we have $\max _{\mathbf{z} \in \Omega_{\delta}^{+}}|M-u(\mathbf{z})|=\delta$ and $d_{1}(\mathbf{x}) \geq d(\mathbf{x})-R_{\delta} \varepsilon$; therefore we obtain

$$
|M-u(\mathbf{x})| \leq \delta e^{-C_{3} \varepsilon^{-1} d(\mathbf{x})}, \quad \mathbf{x} \in \Omega_{\delta}^{+},
$$

where $C_{3}=C_{2}+R_{\delta}$. So (6.2) holds for $\mathrm{x} \in \Omega_{\delta}^{+}$. On the other hand, (6.2) is obviously true for $\mathbf{x} \in \Omega^{+} \backslash \Omega_{\delta}^{+}$when $C_{1}>0$ is chosen properly. The proof for (6.3) is the same.

We recall a recent result by Ghoussoub and Gui GG] (Theorem 1.1, page 482) (see also $[\mathrm{BCN}]$ ).

Theorem 6.3. Suppose that $f \in C^{1}(\mathbf{R})$. If $u$ is a nonconstant solution of

$$
\Delta u(\mathbf{x})+f(u(\mathbf{x}))=0, \quad \mathbf{x}=(x, y) \in \mathbf{R}^{2},
$$

and $u_{x}(\mathbf{x}) \leq 0$ for any $\mathbf{x}=(x, y) \in \mathbf{R}^{2}$, then $u(\mathbf{x})=U\left(\nu \cdot\left(\mathbf{x}-\mathbf{x}_{0}\right)\right)$ for some $\nu, \mathbf{x}_{0} \in \mathbf{R}^{2},|\nu|=1$ and $\nu \neq(0,1)$. If, in addition, $f$ satisfies (f1)-(f4) and (f5a), and $\lim _{x \rightarrow-\infty} u(x, y)=M$ uniformly for all $y$, or $\lim _{x \rightarrow \infty} u(x, y)=m$ uniformly for all $y$, then $\nu=(1,0)$ and $u(\mathbf{x})=U_{1}\left(x-x_{0}\right)$ for some $x_{0} \in \mathbf{R}$, where $U_{1}$ is the unique solution of 2.23.

Finally we reformulate some standard elliptic estimates for the special case of (3.1).

Lemma 6.4. Suppose that $u \in W^{2, p}(\Omega)$, where $p \geq 2, \Omega=(0, a) \times(0, b)$. Then for $\alpha \in(0,1]$ and $\varepsilon>0$, we have

$$
\|u\|_{C^{0}(\bar{\Omega})}+\varepsilon^{\alpha}[u]_{\alpha, \Omega} \leq C\|u\|_{W_{\varepsilon}^{2, p}(\Omega)},
$$


where $C>0$ is independent of $\varepsilon>0$,

$$
\|u\|_{W_{\varepsilon}^{2, p}(\Omega)}=\|u\|_{L^{p}(\Omega)}+\varepsilon\|D u\|_{L^{p}(\Omega)}+\varepsilon^{2}\left\|D^{2} u\right\|_{L^{p}(\Omega)}
$$

and

$$
[u]_{\alpha, \Omega}=\sup _{x, y \in \bar{\Omega}, x \neq y}\left\{\frac{|u(x)-u(y)|}{|x-y|^{\alpha}}\right\} .
$$

Proof. If we rescale $\Omega$ to $\Omega_{\varepsilon}=\varepsilon^{-1} \Omega$ by a change of variables $x=\varepsilon y$, then (6.9) can be obtained from Theorem 7.26 of [GT], except that we need to show that $C$ is independent of $\varepsilon$. From the proofs of Theorems 7.25 and 7.26 of [GT], we know that $C$ depends only on the extension of $u$ from $\Omega_{\varepsilon}$ to $\mathbf{R}^{2}$, and the constant in the extension is independent of $\varepsilon$ since the geometry of all the $\Omega_{\varepsilon}$ are the same.

Lemma 6.5. Suppose that $u$ is a solution of the equation

$$
\varepsilon^{2} \Delta u(\mathbf{x})+g(\mathbf{x})=0, \quad \mathbf{x} \in \Omega, \quad \frac{\partial u}{\partial n}=0 .
$$

Then for $p>1$, and $\varepsilon>0$ small, we have

$$
\|u\|_{W_{\varepsilon}^{2, p}(\Omega)} \leq C\left[\|g\|_{L^{p}(\Omega)}+\|u\|_{L^{p}(\Omega)}\right] .
$$

Proof. We rewrite the equation in (6.10) as $\Delta u+\varepsilon^{-2} g=0$. Then, applying Lemma 2.2 (b) of [NT1, we obtain

$$
\|u\|_{W^{2, p}(\Omega)} \leq C\left[\varepsilon^{-2}\|g\|_{L^{p}(\Omega)}+\|u\|_{L^{p}(\Omega)}\right] .
$$

In particular, we have

$$
\varepsilon^{2}\left\|D^{2} u\right\|_{L^{p}(\Omega)} \leq C\left[\|g\|_{L^{p}(\Omega)}+\|u\|_{L^{p}(\Omega)}\right],
$$

if $\varepsilon>0$ is small. The estimate for $\varepsilon\|D u\|_{L^{p}(\Omega)}$ can be obtained by a modified interpolation theorem as in [GT], Theorem 7.28.

Our main result in this section is the following alternative:

Theorem 6.6. Suppose that $f$ satisfies (f1)-(f4) and (f5a). Then there exists $\bar{\lambda}>$ 0 such that for $\lambda>\bar{\lambda}$, if $(\lambda, u)$ is a solution of (3.1) such that $u_{x} \leq 0$ and $u_{y} \leq 0$ for $\mathbf{x} \in \bar{\Omega}$, then $u$ must be one of the following forms:

1. a constant solution $u=m, \alpha, M$;

2. a semi-trivial solution $u(x, y)=v(x)$, where $v$ is the unique monotone decreasing solution of (2.1);

3. a semi-trivial solution $u(x, y)=v(a y / b)$, where $v$ is the unique monotone decreasing solution of (2.1);

4. $a=b, u$ is a solution such that $\mathcal{N}(u) \cap \partial \Omega=\{(a, 0),(0, b)\}$ and $\mathcal{N}(u)$ intersects both vertices at $45^{\circ}$.

Note that (2.1) has a unique monotone decreasing solution when $\lambda$ is sufficiently large, by the remark at the end of Section 2. The proof of Theorem 6.6 is quite involved; so we sketch the ideas of the proof first, before going on to the details. Since a solution $u$ here is monotone decreasing, then $\mathcal{N}(u)$ is nonempty and it is a monotone curve in $\Omega$. The first step is to prove that $\mathcal{N}(u)$ is very close to a straight line, not only locally, but globally when $\varepsilon=\lambda^{-1 / 2}$ is sufficiently small. This is achieved by using blowup arguments and applying Theorem 6.3 along $\mathcal{N}(u)$. When the nodal line $\mathcal{N}(u)$ intersects the edges of the rectangle, it is orthogonal to the edge because of the Neumann boundary condition; thus $\mathcal{N}(u)$ is close to a 
vertical line when it connects the two horizontal edges. More precisely, letting $\mathcal{N}(u)=\{(q(y), y): y \in[0, b]\}$, we prove that $|q(0)-q(b)|=o(1)$. When at least one end of $\mathcal{N}(u)$ is a vertex of the rectangle, we extend $u$ evenly across the edges; then the extended $\mathcal{N}(u)$ intersect each other at a vertex of the rectangle. From a result on the nodal set of the eigenfunctions of the equation $\Delta u+h(x) u=\lambda u$ by Cheng $[\mathrm{C}$, the nodal lines of $u$ must intersect equiangularly. Thus $\mathcal{N}(u)$ must form a $45^{\circ}$ angle with the neighboring edges, and the other end of $\mathcal{N}(u)$ should also be a vertex since $\mathcal{N}(u)$ is nearly a line, which implies that $\Omega$ is a square.

So it remains to prove that if $\mathcal{N}(u)$ is nearly a vertical line, then $u$ must be the semi-trivial solution $v(x)$. Let $x^{\alpha}$ be the unique point in $(0, a)$ such that $v\left(x^{\alpha}\right)=\alpha$. The second step is to show that $\min \left\{\left|q(0)-x^{\alpha}\right|,\left|q(b)-x^{\alpha}\right|\right\}=o(1)$; that is, the nodal line (or interface) must be near the middle of the rectangle (it is well-known that $\left.\left|x^{\alpha}-(a / 2)\right|=O(\varepsilon|\ln \varepsilon|)\right)$. This part of the proof is based on the comparison method for the corresponding parabolic equation. We prove that if $\mathcal{N}(u)$ is away from $x=x^{\alpha}$, then $u$ cannot be stationary, and a semi-trivial upper solution (or lower solution) will "push" $u$ away. Thus we have shown that the interfaces of $u$ and $v$ are very close to each other, and in fact we can show that $\|u-v\|_{L^{p}(\Omega)}=o(1)$ for $p>0$. The third step is a delicate elliptic estimate to improve the estimates on $u-v$. The proof is a bootstrap argument. Since $w=u-v$ satisfies an equation $\varepsilon^{2} \Delta w+f^{\prime}(\theta) w=0$, we get $\|u-v\|_{W_{\varepsilon}^{2, p}(\Omega)}=o(1)$ from $L^{p}$ estimates, and from that we obtain $\min \left\{\left|q(0)-x^{\alpha}\right|,\left|q(b)-x^{\alpha}\right|\right\}=o(\varepsilon)$, so the interfaces of $u$ and $v$ are both confined in a strip of width $O(\varepsilon)$. Then from the $L^{p}$ estimates and Sobolev embeddings, we finally get

$$
\|u-v\|_{L^{p}(\Omega)} \leq C \varepsilon^{1 / p}\left\|u-v_{1}\right\|_{L^{p}(\Omega)},
$$

which implies $u \equiv v$.

Proof of Theorem 6.6. If $u$ is a monotone decreasing solution, then by the maximum principle, either $u_{x} \equiv 0$ or $u_{x}<0$ for $\mathbf{x} \in \Omega$. Same for $u_{y}$. If $u_{x} \equiv 0$ and $u_{y} \equiv 0$, then we obtain a constant solution. If $u_{x} \equiv 0$ and $u_{y}<0$, then we obtain $v(a y / b)$. If $u_{y} \equiv 0$ and $u_{x}<0$, then we obtain $v(x)$. So from now on, we only consider a solution $u$ satisfying $u_{x}<0$ and $u_{y}<0$. In the proof, we always assume that $u$ is extended to $\mathbf{R}^{2}$ evenly and periodically.

Suppose that there is a sequence of solutions $\left\{\left(\lambda_{j}, u_{j}\right)\right\}$ for $j \geq 1$ such that $\lambda_{j} \rightarrow \infty$ as $j \rightarrow \infty,\left(u_{j}\right)_{x}<0$ and $\left(u_{j}\right)_{y}<0$ for $\mathbf{x} \in \Omega$. Then for any $j \geq$ $1, \mathcal{N}\left(u_{j}\right) \cap \partial \Omega \neq \emptyset$. Indeed, $\mathcal{N}\left(u_{j}\right) \cap \partial \Omega$ is always exactly two points, by the monotonicity of $u_{j}$. Without loss of generality, we assume that $\mathcal{N}\left(u_{j}\right)$ can be written as a graph

$$
\mathcal{N}\left(u_{j}\right)=\left\{\left(q_{j}(y), y\right): y \in\left[0, b_{j}\right]\right\}, \quad \text { where } b_{j} \in(0, b] .
$$

After passing to a subsequence, we consider two cases:

Case 1. $q_{j}(0) \in(0, b)$ for all $j\left(\mathcal{N}\left(u_{j}\right)\right.$ intersects the interior of the edge).

Case 2. $q_{j}(0)=b$ for all $j\left(\mathcal{N}\left(u_{j}\right)\right.$ intersects the boundary of the edge).

We first assume that Case 1 occurs. We prove that $u_{j} \equiv v_{j}$ for $j \geq j_{0}$ for some $j_{0} \in \mathbf{N}$, where $v_{j}$ is the unique monotone decreasing solution of (2.1) with $\lambda=\lambda_{j}$. We select two sequences $\tau_{i} \downarrow m$ and $\varsigma_{i} \uparrow M$ as $i \rightarrow \infty$; then by Lemma 6.1 there 
exist $j_{i}$ and $R_{i}$ such that whenever $j \geq j_{i}$, then

$$
\begin{aligned}
& u_{j}(\mathbf{x}) \leq \tau_{i}, \quad \text { if } \mathbf{x} \in \Omega^{-}\left(u_{j}\right) \text { and } d\left(\mathbf{x}, \mathcal{N}\left(u_{j}\right)\right) \geq R_{i} \varepsilon_{j}, \\
& u_{j}(\mathbf{x}) \geq \varsigma_{i}, \quad \text { if } \mathbf{x} \in \Omega^{+}\left(u_{j}\right) \text { and } d\left(\mathbf{x}, \mathcal{N}\left(u_{j}\right)\right) \geq R_{i} \varepsilon_{j} .
\end{aligned}
$$

We prove our claim in three steps:

Step 1 . We assume that for any $i, q_{j}(0)<b-\varepsilon_{j} R_{i}$ for all $j$. We prove that $b_{j}=b$ for $j \geq j_{1}$ and, for $y \in[0, b]$,

$$
q_{j}(y)=q_{j}(0)+o(1), \quad q_{j}^{\prime}(y)=o(1), \quad \text { as } j \rightarrow \infty .
$$

Moreover, for each $i$ and $y_{0} \in[0, b], j \geq j_{i}$,

$$
\left\|u_{j}(\mathbf{x})-U_{1}\left(\frac{\mathbf{x}-\mathbf{x}_{j}^{y_{0}}}{\varepsilon_{j}}\right)\right\|_{C_{j}^{2}\left(\overline{\Omega_{i, j}^{y_{0}}}\right)} \rightarrow 0, \quad \text { as } j \rightarrow \infty,
$$

where $U_{1}$ is the unique solution of (2.23),

$$
\|v\|_{C_{j}^{2}(\bar{\Omega})}=\|v\|_{C^{0}(\bar{\Omega})}+\varepsilon_{j}\|D v\|_{C^{0}(\bar{\Omega})}+\varepsilon_{j}^{2}\left\|D^{2} v\right\|_{C^{0}(\bar{\Omega})},
$$

$\mathbf{x}_{j}^{y_{0}}=\left(q_{j}\left(y_{0}\right), y_{0}\right)$ and

$$
\Omega_{i, j}^{y_{0}}=\left\{(x, y):\left|y-y_{0}\right|<\frac{1}{2} R_{i} \varepsilon_{j},\left|x-q_{j}\left(y_{0}\right)\right|<\frac{1}{2} R_{i} \varepsilon_{j}\right\} .
$$

In particular, we define a sequence of tubular neighborhoods around $\mathcal{N}\left(u_{j}\right)$ :

$$
T_{i, j}=\left\{\mathbf{x} \in \Omega: \operatorname{dist}\left(\mathbf{x}, \mathcal{N}\left(u_{j}\right)\right) \leq \frac{1}{2} \varepsilon_{j} R_{i}\right\}
$$

then from 6.15 we have

$$
\varepsilon_{j}\left\|\left(u_{j}\right)_{y}\right\|_{C^{0}\left(\overline{T_{i, j}}\right)}+\varepsilon_{j}^{2}\left\|\left(u_{j}\right)_{x y}\right\|_{C^{0}\left(\overline{T_{i, j}}\right)}+\varepsilon_{j}^{2}\left\|\left(u_{j}\right)_{y y}\right\|_{C^{0}\left(\overline{T_{i, j}}\right)} \rightarrow 0,
$$

as $j \rightarrow \infty$, since $U_{1}$ only depends on $x$.

Proof of Step 1. (A). Define regions

$$
\widetilde{\Omega_{i, j}^{0}}=\left\{(x, y) \in \Omega:|y|<R_{i} \varepsilon_{j},\left|x-q_{j}(0)\right|<R_{i} \varepsilon_{j}\right\} .
$$

We prove that for each fixed $i$, there exists a subsequence of $\left\{u_{j}\right\}$ (which is still denoted by $\left\{u_{j}\right\}$ to simplify the notation) such that

$$
\left\|u_{j}(x, y)-U_{1}\left(\frac{x-q_{j}(0)}{\varepsilon_{j}}\right)\right\|_{C_{j}^{2}\left(\widetilde{\Omega_{i, j}^{0}}\right)} \rightarrow 0, \quad \text { as } j \rightarrow \infty,
$$

and

$$
q_{j}\left(r \varepsilon_{j}\right)=q_{j}(0)+o\left(\varepsilon_{j}\right), \quad q_{j}^{\prime}\left(r \varepsilon_{j}\right)=o(1),
$$

uniformly for $r \in\left[0, R_{i}\right]$, as $j \rightarrow \infty$.

Define $v_{j}(\mathbf{z})=u_{j}\left(\mathbf{x}_{j}^{0}+\varepsilon_{j} \mathbf{z}\right)$ for $\mathbf{z} \in S\left(2 R_{i}\right)$ where $S(r)$ is defined as

$$
S(r)=\left\{\left(z_{1}, z_{2}\right):-r<z_{1}<r,-r<z_{2}<r\right\} .
$$

Note that $v_{j}$ is well defined because of our assumption that $q_{j}(0)<b-\varepsilon_{j} R_{i}$. Clearly $v_{j}$ satisfies the equation

$$
\Delta v_{j}(\mathbf{z})+f\left(v_{j}(\mathbf{z})\right)=0, \quad \mathbf{z} \in S\left(2 R_{i}\right) .
$$

We claim that $\left\{v_{j}\right\}$ is bounded in $C^{2, \alpha}\left(\overline{S\left(R_{i}\right)}\right)$ for any $\alpha \in(0,1)$. From the maximum principle, we know that $m<u_{j}(\mathbf{x})<M$ for $\mathbf{x} \in \bar{\Omega}$. Thus $\left\|v_{j}\right\|_{C^{0}\left(\overline{S\left(2 R_{i}\right)}\right)} \leq C_{1}$ 
and $|f(u)| \leq C_{2}$ for all $u \in[m, M]$, and then, by the interior Schauder estimates of the elliptic equations, we have

$$
\left\|v_{j}\right\|_{C^{2, \alpha}\left(\overline{S\left(R_{i}\right)}\right)} \leq C_{3}\left(\left\|v_{j}\right\|_{C^{0}\left(\overline{S\left(2 R_{i}\right)}\right)}+C_{2}\right) \leq C_{4} .
$$

Therefore $\left\{v_{j}\right\}$ is a relatively compact set in $C^{2}\left(\overline{S\left(R_{i}\right)}\right)$, and by a diagonal process, we obtain a subsequence (still denoted by $\left\{v_{j}\right\}$ ) such that

$$
v_{j} \rightarrow v_{\infty} \quad \text { in } C_{l o c}^{2}\left(\mathbf{R}^{2}\right) \text {. }
$$

The limit function $v_{\infty} \in C^{2}\left(\mathbf{R}^{2}\right)$ satisfies

$$
\Delta v_{\infty}(\mathbf{z})+f\left(v_{\infty}(\mathbf{z})\right)=0, \quad \mathbf{z} \in \mathbf{R}^{2},
$$

and $\left(v_{\infty}\right)_{z_{1}} \leq 0$ for all $\mathbf{z}$ since $\left(v_{j}\right)_{z_{1}}<0$. By the definition of $R_{i}$, we have $v_{j}\left(R_{i}, z_{2}\right) \leq \tau_{i}$ for $j \geq j_{1}$ and $\left|z_{2}\right| \leq R_{i}$. Indeed, for any $z_{2}>R_{i}$, we still have $v_{j}\left(R_{i}, z_{2}\right) \leq \tau_{i}$ since $q_{j}^{\prime}(y)<0$ and $q_{j}(y) \leq q_{j}(0)$; and

$$
\operatorname{dist}\left(\left(q_{j}(0)+\varepsilon_{j} R_{i}, \varepsilon_{j} z_{2}\right), \mathcal{N}\left(u_{j}\right)\right) \geq \varepsilon_{j} R_{i} .
$$

Thus $v_{\infty}\left(R_{i}, z_{2}\right)=\lim _{j \rightarrow \infty} v_{j}\left(R_{i}, z_{2}\right) \leq \tau_{i}$, which implies $v_{\infty}\left(z_{1}, z_{2}\right) \rightarrow m$ uniformly for $z_{2}$ as $z_{1} \rightarrow \infty$. Therefore, by Theorem 6.3 we conclude that either $v_{\infty}\left(z_{1}, z_{2}\right)=$ $U_{1}\left(z_{1}-z_{0}\right)$ for some $z_{0} \in \mathbf{R}$, or $v_{\infty} \equiv m$. But $v_{\infty}(0,0)=\alpha$, and so $v_{\infty}(\mathbf{z})=U_{1}\left(z_{1}\right)$.

To prove [6.22), we define $Q_{j}\left(z_{2}\right)=\varepsilon_{j}^{-1}\left[q_{j}\left(\varepsilon_{j} z_{2}\right)-q_{j}(0)\right]$. Then $Q_{j}$ satisfies $v_{j}\left(Q_{j}\left(z_{2}\right), z_{2}\right)=\alpha$. From the convergence of $v_{j}$, we obtain that $Q_{j} \rightarrow Q_{\infty}$ in $C_{l o c}^{2}(\mathbf{R})$, and $v_{\infty}\left(Q_{\infty}\left(z_{2}\right), z_{2}\right)=\alpha$. Then $Q_{\infty}\left(z_{2}\right) \equiv 0$, and consequently we obtain (6.22).

(B). We prove that for each fixed $i$, and $y_{0} \in\left(0, b_{j}\right)$, there exists a subsequence of $\left\{u_{j}\right\}$ (still denoted by $\left\{u_{j}\right\}$ ) such that

$$
\left\|u_{j}(\mathbf{x})-U_{1}\left(\frac{\nu_{j}\left(y_{0}\right) \cdot\left(\mathbf{x}-\mathbf{x}_{j}^{y_{0}}\right)}{\varepsilon_{j}}\right)\right\|_{C_{j}^{2}\left(\overline{\Omega_{i, j}^{y_{0}}}\right)} \rightarrow 0, \quad \text { as } j \rightarrow \infty,
$$

where

$$
\begin{gathered}
\nu_{j}\left(y_{0}\right)=\frac{\left(1,-q_{j}^{\prime}\left(y_{0}\right)\right)}{\sqrt{1+\left[q_{j}^{\prime}\left(y_{0}\right)\right]^{2}}}, \\
\widetilde{\Omega_{i, j}^{y_{0}}}=\left\{\theta_{j}\left(y_{0}\right) \cdot(x, y)^{T}:\left|y-y_{0}\right|<R_{i} \varepsilon_{j},\left|x-q_{j}\left(y_{0}\right)\right|<R_{i} \varepsilon_{j}\right\}, \\
\theta_{j}\left(y_{0}\right)=\frac{1}{\sqrt{1+\left[q_{j}^{\prime}\left(y_{0}\right)\right]^{2}}}\left(\begin{array}{cc}
1 & -q_{j}^{\prime}\left(y_{0}\right) \\
q_{j}^{\prime}\left(y_{0}\right) & 1
\end{array}\right),
\end{gathered}
$$

and

$$
q_{j}\left(y_{0}+r \varepsilon_{j}\right)=q_{j}\left(y_{0}\right)+o\left(\varepsilon_{j}\right), \quad q_{j}^{\prime}\left(y_{0}+r \varepsilon_{j}\right)=q_{j}^{\prime}\left(y_{0}\right)+o(1),
$$

uniformly for $r \in\left[-R_{i}, R_{i}\right]$, as $j \rightarrow \infty$.

The idea is similar to $(\mathrm{A})$, and we blow up the solutions $u_{j}$ at $\left(q_{j}(y), y\right)$. We define $v_{j}^{y_{0}}(\mathbf{z})=u_{j}\left(\mathbf{x}_{j}^{y_{0}}+\varepsilon_{j} \theta_{j}\left(y_{0}\right) \cdot \mathbf{z}\right)$, where $\theta_{j}\left(y_{0}\right)$ is the rotation defined in (6.31), and $\mathbf{z} \in S\left(2 R_{i}\right)$. Since $\Delta$ is invariant under rotation, then we can apply the same argument as in (A) to conclude that $v_{j}^{y_{0}} \rightarrow v_{\infty}^{y_{0}}$ in $C_{l o c}^{2}\left(\mathbf{R}^{2}\right)$ and $v_{\infty}^{y_{0}}$ satisfies the equation (6.27). Since

$$
\frac{\partial v_{j}^{y_{0}}\left(z_{1}, z_{2}\right)}{\partial z_{1}}=\varepsilon_{j}^{-1} \nu_{j}\left(y_{0}\right) \cdot\left(\begin{array}{c}
\left(u_{j}\right)_{x} \\
\left(u_{j}\right)_{y}
\end{array}\right)
$$


and $q_{j}^{\prime}\left(y_{0}\right)<0$, then $\partial v_{j}^{y_{0}}\left(z_{1}, z_{2}\right) / \partial z_{1}<0$, which implies $\partial v_{\infty}^{y_{0}}\left(z_{1}, z_{2}\right) / \partial z_{1} \leq 0$. Since $v_{j}^{y_{0}}(0,0)=\alpha$, by Theorem [6.3, $v_{\infty}^{y_{0}}$ is either the constant $\alpha$ or $U_{1}\left(z_{1}\right)$. We claim that it cannot be the constant $\alpha$. Since $\mathcal{N}\left(u_{j}\right)$ is a monotone curve, and by a simple geometric observation, we can find that the distance from at least two of the vertices of the square $\widetilde{\Omega_{i, j}^{y_{0}}}$ to $\mathcal{N}\left(u_{j}\right)$ is not less than $\varepsilon_{j} R_{i}$, which means there is a subsequence of $\left\{v_{j}\right\}$ (still denoted the same) which satisfies $v_{j}\left(\mathbf{z}_{j}\right) \leq \tau_{i}$ or $v_{j}\left(\mathbf{z}_{j}\right) \geq \varsigma_{i}$, with $\mathbf{z}_{j}$ being one such vertex. Thus the limit function cannot be the constant $\alpha$, and $v_{\infty}^{y_{0}}=U_{1}\left(z_{1}\right)$. Finally, the estimates on $q_{j}$ and $q_{j}^{\prime}$ can also be proved similarly to $(\mathrm{A})$.

(C). We prove the statements in Step 1. Since the convergence in (A) and (B) holds for any subsequence, and the limit is unique, then the convergence holds for the whole sequence. In particular, the estimates for $q_{j}$ and $q_{j}^{\prime}$ hold for any $j$ large enough. Thus for fixed $R>0$ and $\varepsilon_{j}$, we can choose a sequence $\left\{y_{i}: 0 \leq i \leq N=\right.$ $N(R, j)\}$ such that $y_{0}=0, y_{N}=b,\left|y_{i}-y_{i+1}\right| \leq R \varepsilon_{j}$ (clearly we can always make a selection so that $N(R, j) \leq C R^{-1} \varepsilon_{j}^{-1}$ for some constant $C$ ). Then (6.14) can be obtained by (6.32) if we fix $R$. In particular, we obtain $b_{j}=b$, and so the nodal line must intersect the opposite edges of $\Omega$. The convergence in 6.15 holds since $q_{j}^{\prime} \rightarrow 0, \nu_{j}\left(y_{0}\right) \rightarrow(1,0)$ and $\theta_{j}\left(y_{0}\right) \rightarrow I$ as $j \rightarrow \infty$.

Step 2. We prove that

$$
\left|q_{j}(0)-x_{j}^{\alpha}\right| \rightarrow 0, \quad \text { as } j \rightarrow \infty,
$$

where $x_{j}^{\alpha}$ is the unique point such that $v_{j}\left(x_{j}^{\alpha}\right)=\alpha$. On the other hand, since $\left|x_{j}^{\alpha}-(a / 2)\right|=O(|\varepsilon \ln \varepsilon|)$, then

$$
\lim _{j \rightarrow \infty} q_{j}(0)=\lim _{j \rightarrow \infty} q_{j}(b)=\frac{a}{2} .
$$

Proof of Step 2. Suppose this is not true. Then without loss of generality we assume that there exists a subsequence of $\left\{u_{j}\right\}$ (still denoted by $\left\{u_{j}\right\}$ ) such that $q_{j}(0)-x_{j}^{\alpha} \geq \eta>0$. We claim that for sufficiently large $j, u_{j}(x, b)>v_{j}(x)$. To prove the claim, we choose $\delta>0$ such that $f^{\prime}(u)<0$ for $u \in(m, m+\delta) \cup(M-\delta, M)$. Since $v_{j}$ is decreasing and $v_{j}(0) \rightarrow M, v_{j}(a) \rightarrow m$ as $j \rightarrow \infty$, then there exist $0<x_{j}^{1}<x_{j}^{\alpha}<x_{j}^{2}<a$ such that $v_{j}\left(x_{j}^{1}\right)=M-\delta$ and $v_{j}\left(x_{j}^{2}\right)=m+\delta$. From Lemma [6.1, $\max \left(\left|x_{j}^{1}-x_{j}^{\alpha}\right|,\left|x_{j}^{2}-x_{j}^{\alpha}\right|\right)=O\left(\varepsilon_{j}\right)$. On the other hand, also from Lemma 6.1 there exists $K_{\delta}>0$ such that when $0<x<q_{j}(b)-K_{\delta} \varepsilon_{j}$, we have $u_{j}(x, y)>M-\delta$. Since $q_{j}(0)-x_{j}^{\alpha} \geq \eta>0$, then there exists $j_{2}>0$ such that when $j \geq j_{2}, x_{j}^{2}<q_{j}(b)-K_{\delta} \varepsilon_{j}$. For $x \in\left(x_{j}^{1}, q_{j}(b)-K_{\delta} \varepsilon_{j}\right), u_{j}(x, b)>M-\delta>v_{j}(x)$. Suppose that $u_{j}(x, b)-v_{j}(x)$ reaches a negative minimum at $x_{j}^{*}$; then $x_{j}^{*}$ belongs to either $\left[0, x_{j}^{1}\right]$ or $\left[q_{j}(b)-K_{\delta} \varepsilon_{j}, a\right]$. We recall that $\left(u_{j}\right)_{y y}(x, b) \geq 0$ for $x \in[0, a]$; thus $w_{j}(x)=u_{j}(x, b)-v_{j}(x)$ satisfies the equation

$$
\left(w_{j}\right)_{x x}+\lambda_{j} \frac{f\left(u_{j}(x, b)\right)-f\left(v_{j}(x)\right)}{u_{j}(x, b)-v_{j}(x)} w_{j}=-\left(u_{j}\right)_{y y}(x, b) \leq 0 .
$$

If $x_{j}^{*} \in\left[0, x_{j}^{1}\right]$ and both $u_{j}\left(x_{j}^{*}, b\right)$ and $v_{j}\left(x_{j}^{*}\right)$ are greater than $M-\delta$, then

$$
\left[f\left(u_{j}\left(x_{j}^{*}, b\right)\right)-f\left(v_{j}\left(x_{j}^{*}\right)\right)\right] /\left[u_{j}\left(x_{j}^{*}, b\right)-v_{j}\left(x_{j}^{*}\right)\right]<0
$$


and $w_{j}\left(x_{j}^{*}\right)<0$; thus $\left(w_{j}\right)_{x x}\left(x_{j}^{*}\right)<0$, which contradicts that $x_{j}^{*}$ is a local minimum. If $x_{j}^{*} \in\left[q_{j}(b)-K_{\delta} \varepsilon_{j}, a\right]$ and $u_{j}\left(x_{j}^{*}, b\right)<v_{j}\left(x_{j}^{*}\right) \leq m+\delta$, then we can get a contradiction similarly. Therefore $u_{j}(x, b)>v_{j}(x)$ for sufficiently large $j$. In particular, $u_{j}(x, y) \geq u_{j}(x, b)>v_{j}(x)$ for all $(x, y) \in \Omega$.

We consider the parabolic equation corresponding to (3.1):

$$
\begin{cases}u_{t}=\Delta u+\lambda f(u), & (t, \mathbf{x}) \in(0, \infty) \times \Omega, \\ \frac{\partial u}{\partial n}=0, & (t, \mathbf{x}) \in(0, \infty) \times \partial \Omega, \\ u(0, \mathbf{x})=u_{0}(\mathbf{x}), & \mathbf{x} \in \Omega .\end{cases}
$$

Let $U_{j}(t, \mathbf{x})$ be the solution of (6.37) with $u_{0}(\mathbf{x})=V_{j}(x)$ and $\lambda=\lambda_{j}$, where $V_{j}(x)$ is a function defined on $[0, a]$, satisfying $V_{j}^{\prime}(0)=V_{j}^{\prime}(a)=0, V_{j}^{\prime}(x)<0$ for $x \in(0, a)$, and $v_{j}(x)<V_{j}(x)<u_{j}(x, b) \leq u_{j}(x, y)$. From the uniqueness of the solution we get $U_{j}(t, x, y) \equiv U_{j}(t, x)$, which is the solution of

$$
\begin{cases}u_{t}=u_{x x}+\lambda_{j} f(u), & (t, x) \in(0, \infty) \times(0, a), \\ u_{x}(t, 0)=u_{x}(t, a)=0, & u(0, x)=V_{j}(x), \quad x \in(0, a) .\end{cases}
$$

It is well-known (see, for example, Henry $[\mathrm{H}]$ ) that $U_{j}(t, x)$ exists globally and the $\omega$-limit set $\omega\left(U_{j}\right)$ of $U_{j}$ is one equilibrium solution of (6.38). On the other hand, from the nonincreasing property (with respect to $t$ ) of the lap number of solutions to (6.38) (here the lap number is defined as the sign-changing of $U_{j}(t, \cdot)-\alpha$, see, for example, Matano [M2] $), \omega\left(U_{j}\right)$ must be one of the following: $m, M, v_{j}(x)$ and $v_{j}(a-x)$, the only equilibrium solutions with lap number less than or equal to 1. First, from our assumptions, $U_{j}(0, x)>v_{j}(x)$, and so $U_{j}(t, x)>v_{j}(x)$ for all $t>0$ by the maximum principle. If $\omega\left(U_{j}\right)$ is $v_{j}(x)$ or $v_{j}(a-x)$, say $v_{j}(x)$, then $V_{j}(\cdot)$ belongs to the stable manifold of $v_{j}(\cdot)$, but from a result of Brunovský and Fiedler [BrF] (page 190, Theorem 3.2), the lap number of $V_{j}-v_{j}$ is no less than the dimension of the unstable manifold of $v_{j}$, which is 1 in this case. But the lap number of $V_{j}-v_{j}$ is zero, a contradiction. $\omega\left(U_{j}\right)$ cannot be $m$ either, since $U_{j}(t, x)>v_{j}(x)$ for all $t>0$. Hence $\omega\left(U_{j}\right)=\{M\}$.

Now we reach a contradiction by the comparison principle. In fact, $U_{j}(0, x, y)=$ $V_{j}(x)<u_{j}(x, y)$, so $U_{j}(t, x, y) \leq u_{j}(x, y)$ by the comparison principle. But on the other hand, $U_{j}(t, x) \rightarrow M$ when $t \rightarrow \infty$, which is a contradiction.

We note that the proof of Step 2 did not need the assumption $q_{j}(0)<b-\varepsilon_{j} R_{i}$ in Step 1. Indeed, if this assumption is not satisfied here, then from Step 1 we must have $q_{j}(b)=a+o(1)$ or $q_{j}(0)=o(1)$, but in either case we can use the proof of Step 2 to reach a contradiction.

Step 3. We prove that $u_{j} \equiv v_{j}$.

Proof of Step 3. (A). We prove

$$
\lim _{j \rightarrow \infty}\left\|u_{j}-v_{j}\right\|_{W_{\varepsilon_{j}}^{2, p}(\Omega)}=0,
$$

where $0<p<\infty$.

First we prove that

$$
\lim _{j \rightarrow \infty}\left\|u_{j}-v_{j}\right\|_{L^{p}(\Omega)}=0, \quad 0<p<\infty .
$$


Recall that in the proof of Lemma 6.2, we chose $\delta>0$ such that $f^{\prime}(u)<0$ for $u \in(m, m+\delta) \cup(M-\delta, M)$. By Lemma 6.1 there exists $R_{\delta}>0$ such that

$$
\begin{aligned}
& u_{j}(\mathbf{x}) \leq m+\delta, \quad \text { if } \mathbf{x} \in \Omega^{-}\left(u_{j}\right) \text { and } d\left(\mathbf{x}, \mathcal{N}\left(u_{j}\right)\right) \geq R_{\delta} \varepsilon_{j}, \\
& u_{j}(\mathbf{x}) \geq M-\delta, \quad \text { if } \mathbf{x} \in \Omega^{+}\left(u_{j}\right) \text { and } d\left(\mathbf{x}, \mathcal{N}\left(u_{j}\right)\right) \geq R_{\delta} \varepsilon_{j}, \\
& v_{j}(\mathbf{x}) \leq m+\delta, \quad \text { if } \mathbf{x} \in \Omega^{-}\left(v_{j}\right) \text { and } d\left(\mathbf{x}, \mathcal{N}\left(v_{j}\right)\right) \geq R_{\delta} \varepsilon_{j}, \\
& v_{j}(\mathbf{x}) \geq M-\delta, \quad \text { if } \mathbf{x} \in \Omega^{+}\left(v_{j}\right) \text { and } d\left(\mathbf{x}, \mathcal{N}\left(v_{j}\right)\right) \geq R_{\delta} \varepsilon_{j} .
\end{aligned}
$$

Since $\mathcal{N}\left(v_{j}\right)$ is a line segment $\left\{\left(x_{j}^{\alpha}, y\right): 0 \leq y \leq b\right\}$, and $\mathcal{N}\left(u_{j}\right)$ is a monotone curve $\left\{\left(q_{j}(y), y\right): 0 \leq y \leq b\right\}$, then we obtain that

$$
\begin{aligned}
& u_{j}(\mathbf{x}) \leq m+\delta, \quad v_{j}(\mathbf{x}) \leq m+\delta, \quad \text { if } 0 \leq x \leq L_{j} \\
& u_{j}(\mathbf{x}) \geq M-\delta, \quad v_{j}(\mathbf{x}) \geq M-\delta, \quad \text { if } R_{j} \leq x \leq a
\end{aligned}
$$

where

$$
\begin{aligned}
& L_{j}=\min \left(x_{j}^{\alpha}-R_{\delta} \varepsilon_{j}, q_{j}(b)-R_{\delta} \varepsilon_{j}\right), \\
& R_{j}=\max \left(x_{j}^{\alpha}+R_{\delta} \varepsilon_{j}, q_{j}(0)+R_{\delta} \varepsilon_{j}\right) .
\end{aligned}
$$

We define

$$
\begin{gathered}
\Omega_{j}^{+}=\left\{(x, y) \in \Omega: 0 \leq x \leq L_{j}\right\}, \Omega_{j}^{-}=\left\{(x, y) \in \Omega: R_{j} \leq x \leq a\right\}, \\
\Omega_{j}^{0}=\left\{(x, y) \in \Omega: L_{j} \leq x \leq R_{j}\right\} .
\end{gathered}
$$

From (6.14) and (6.34), we could see that $R_{j}-L_{j}=o(1)$ as $j \rightarrow \infty$, and by the maximum principle, $m<u_{j}(\mathbf{x}), v_{j}(\mathbf{x})<M$. Thus

$$
\int_{\Omega_{j}^{0}}\left|u_{j}-v_{j}\right|^{p} d \mathbf{x} \leq(M-m)^{p} \cdot o(1) .
$$

In the subdomain $\Omega_{j}^{+}$, from Lemma 6.2, we have

$$
|M-u| \leq C_{1} e^{-C_{2} \varepsilon_{j}^{-1}\left|x-L_{j}\right|}, \quad x \in \Omega_{j}^{+},
$$

for $u=u_{j}$ or $v_{j}$. Then

$$
\int_{\Omega_{j}^{+}}\left|u_{j}-v_{j}\right|^{p} d \mathbf{x} \leq C \int_{\Omega_{j}^{+}} e^{-C_{2} p \varepsilon_{j}^{-1}\left|x-L_{j}\right|} d \mathbf{x}=O\left(\varepsilon_{j}\right) .
$$

A similar estimate holds for $\Omega_{j}^{-}$. Therefore,

$$
\left\|u_{j}-v_{j}\right\|_{L^{p}(\Omega)} \leq o\left(\varepsilon_{j}^{1 / p}\right)+o(1)=o(1) .
$$

Since $u_{j}-v_{j}$ satisfies the equation

$$
\begin{cases}\varepsilon_{j}^{2} \Delta\left(u_{j}-v_{j}\right)+\left[f\left(u_{j}\right)-f\left(v_{j}\right)\right]=0, & x \in \Omega, \\ \frac{\partial\left(u_{j}-v_{j}\right)}{\partial n}=0, & x \in \partial \Omega,\end{cases}
$$

then from Lemma 6.5 and $\left|f^{\prime}(u)\right| \leq C$ for $u \in(m, M)$, we obtain (6.39).

(B). We prove that

$$
\max \left(\left|q_{j}(b)-x_{j}^{\alpha}\right|,\left|q_{j}(0)-x_{j}^{\alpha}\right|\right)=O\left(\varepsilon_{j}\right), \quad \text { as } j \rightarrow \infty,
$$

which also implies

$$
\left|q_{j}(b)-q_{j}(0)\right|=O\left(\varepsilon_{j}\right), \quad \text { as } j \rightarrow \infty \text {. }
$$


Suppose it is not true; without loss of generality, we assume that there exists a subsequence of $\left\{u_{j}\right\}$ (still denoted by $\left\{u_{j}\right\}$ ) such that

$$
\frac{q_{j}(0)-x_{j}^{\alpha}}{\varepsilon_{j}} \geq \widetilde{R}_{j}
$$

for a sequence $\widetilde{R}_{j} \rightarrow \infty$ as $j \rightarrow \infty$. Again let $\delta$ and $R_{\delta}$ be the same as in (A). Then for $(x, y) \in \Omega_{j, \delta}^{0} \equiv\left\{(x, y):\left|x-q_{j}(0)\right|<\varepsilon_{j} R_{\delta}, 0<y<\varepsilon_{j} R_{\delta}\right\}$, we choose $j$ large enough so that $\widetilde{R}_{j} \geq 3 R_{\delta}$. Then from the proof in (A), we obtain

$$
\left|v_{j}(x, y)-M\right| \leq C_{1} e^{-C_{2} \varepsilon_{j}^{-1}\left|x-x_{j}^{\alpha}\right|} \leq C_{1} e^{-C_{2} \varepsilon_{j}^{-1} R_{\delta}}, \quad(x, y) \in \Omega_{j, \delta}^{0} .
$$

On the other hand, by (6.21),

$$
\left\|u_{j}(x, y)-U_{1}\left(\frac{x-q_{j}(0)}{\varepsilon_{j}}\right)\right\|_{C_{j}^{2}\left(\overline{\Omega_{j, \delta}^{0}}\right)} \rightarrow 0, \quad \text { as } j \rightarrow \infty .
$$

From (6.47) and (6.48), we get

$$
\begin{aligned}
& \left\|u_{j}-v_{j}\right\| \\
& \quad \geq\left\|U_{1}\left(\frac{x-q_{j}(0)}{\varepsilon_{j}}\right)-M\right\|-\left\|v_{j}-M\right\|-\left\|U_{1}\left(\frac{x-q_{j}(0)}{\varepsilon_{j}}\right)-u_{j}\right\| \\
& \geq\left\|U_{1}\left(\frac{x-q_{j}(0)}{\varepsilon_{j}}\right)-M\right\|-O\left(e^{-C_{2} \varepsilon_{j}^{-1} R_{\delta}}\right)-o(1),
\end{aligned}
$$

where the norm $\|\cdot\|$ is $\|\cdot\|_{W_{\varepsilon_{j}}^{2, p}\left(\Omega_{j, \delta}^{0}\right)}$. Moreover,

$$
\begin{aligned}
& \left\|U_{1}\left(\frac{x-q_{j}(0)}{\varepsilon_{j}}\right)-M\right\|_{W_{\varepsilon_{j}, p}^{2, p}\left(\Omega_{j, \delta}^{0}\right)} \\
& \quad=\left\|U_{1}-M\right\|_{L^{p}}+\varepsilon_{j}\left\|D U_{1}\right\|_{L^{p}}+\varepsilon_{j}^{2}\left\|D^{2} U_{1}\right\|_{L^{p}} \\
& \quad=O\left(\varepsilon_{j}\right)+R_{\delta}\left[\left(\int_{-R_{\delta}}^{R_{\delta}}\left[U_{1}^{\prime}\left(z_{1}\right)\right]^{p} d z_{1}\right)^{1 / p}+\left(\int_{-R_{\delta}}^{R_{\delta}}\left[U_{1}^{\prime \prime}\left(z_{1}\right)\right]^{p} d z_{1}\right)^{1 / p}\right] .
\end{aligned}
$$

Then in (6.49) and (6.50), we reach a contradiction with (6.39). So (6.44) is true.

(C). We prove that

$$
\begin{gathered}
\max \left(\left|q_{j}(b)-x_{j}^{\alpha}\right|,\left|q_{j}(0)-x_{j}^{\alpha}\right|\right)=o\left(\varepsilon_{j}\right), \quad \text { as } j \rightarrow \infty \\
\left\|u_{j}(x, y)-U_{1}\left(\frac{x-x_{j}^{\alpha}}{\varepsilon_{j}}\right)\right\|_{C_{j}^{2}\left(\overline{\Omega_{j, R}}\right)} \rightarrow 0, \quad \text { as } j \rightarrow \infty
\end{gathered}
$$

and

$$
\left\|u_{j}-v_{j}\right\|_{C_{j}^{2}\left(\overline{\Omega_{j, R}}\right)} \rightarrow 0, \quad \text { as } j \rightarrow \infty
$$

for $R>0$, where

$$
\Omega_{j, R}=\left\{(x, y):\left|x-x_{j}^{\alpha}\right|<\varepsilon_{j} R, 0<y<b\right\} .
$$

Suppose (6.51) is not true. Then there is a subsequence of $\left\{u_{j}\right\}$ (still denoted by $\left.\left\{u_{j}\right\}\right)$ such that

$$
\frac{q_{j}(0)-x_{j}^{\alpha}}{\varepsilon_{j}} \geq \eta_{1}>0 .
$$


On the other hand, from (6.44), the sequence $\varepsilon_{j}^{-1}\left[q_{j}(0)-x_{j}^{\alpha}\right]$ is also bounded, so by taking a further subsequence, we assume that

$$
\lim _{j \rightarrow \infty} \frac{q_{j}(0)-x_{j}^{\alpha}}{\varepsilon_{j}}=\eta>0 .
$$

We choose $R>2 \eta$, and define

$$
\Omega_{j, R}^{0}=\left\{(x, y):\left|x-x_{j}^{\alpha}\right|<\varepsilon_{j} R, 0<y<\varepsilon_{j} R\right\} .
$$

From the proof in Step 1, we have

$$
\left\|u_{j}(x, y)-U_{1}\left(\frac{x-q_{j}(0)}{\varepsilon_{j}}\right)\right\|_{C_{j}^{2}\left(\overline{\Omega_{j, R}^{0}}\right)} \rightarrow 0, \text { as } j \rightarrow \infty,
$$

and

$$
\left\|v_{j}(x, y)-U_{1}\left(\frac{x-x_{j}^{\alpha}}{\varepsilon_{j}}\right)\right\|_{C_{j}^{2}\left(\overline{\Omega_{j, R}^{0}}\right)} \rightarrow 0, \quad \text { as } j \rightarrow \infty .
$$

Then from (6.58), (6.59) and the same argument as in (6.49), we reach a contradiction with (6.39), since

$$
\begin{aligned}
& \left\|U_{1}\left(\frac{x-q_{j}(0)}{\varepsilon_{j}}\right)-U_{1}\left(\frac{x-x_{j}^{\alpha}}{\varepsilon_{j}}\right)\right\|_{W_{\varepsilon}^{2, p}\left(\Omega_{j, R}^{0}\right)} \\
\geq & R_{\delta}\left(\int_{-\eta}^{\eta}\left[U_{1}^{\prime}\left(z_{1}\right)-U_{1}^{\prime}\left(z_{1}-\eta\right)\right]^{p} d z_{1}\right)^{1 / p} \\
& +R_{\delta}\left(\int_{-\eta}^{\eta}\left[U_{1}^{\prime \prime}\left(z_{1}\right)-U_{1}^{\prime \prime}\left(z_{1}-\eta\right)\right]^{p} d z_{1}\right)^{1 / p}+o(1) .
\end{aligned}
$$

Therefore (6.51) is true. From (6.58), (6.60) and (6.51), we obtain

$$
\left\|u_{j}(x, y)-U_{1}\left(\frac{x-x_{j}^{\alpha}}{\varepsilon_{j}}\right)\right\|_{C_{j}^{2}\left(\overline{\Omega_{j, R}^{0}}\right)} \rightarrow 0, \quad \text { as } j \rightarrow \infty .
$$

And obviously (6.61) can also be obtained if we replace the subdomain $\Omega_{j, R}^{0}$ by any $\Omega_{j, R}^{y}=\Omega_{j, R}^{0}+y$ for $y \in[0, b]$. So (6.52) is also true.

(D). As in (A), we use the notation $L_{j}, R_{j}, \Omega_{j}^{ \pm}$and $\Omega_{j}^{0}$. Then from (C), we obtain

$$
\int_{\Omega_{j}^{0}}\left|u_{j}-v_{j}\right|^{p} d \mathbf{x} \leq C \varepsilon_{j}\left\|u_{j}-v_{j}\right\|_{C^{0}(\bar{\Omega})}^{p}
$$

For $\mathbf{x} \in \Omega_{j}^{+}$, using the proof of Lemma 6.2 we have

$$
\left|u_{j}(\mathbf{x})-v_{j}(\mathbf{x})\right| \leq \max _{\mathbf{z} \in \overline{\Omega_{j}^{+}}}\left|u_{j}(\mathbf{z})-v_{j}(\mathbf{z})\right| e^{-C_{2} \varepsilon_{j}^{-1}\left|x-L_{j}\right|} .
$$

Thus

$$
\int_{\Omega_{j}^{+}}\left|u_{j}-v_{j}\right|^{p} d \mathbf{x} \leq C \varepsilon_{j}|| u_{j}-v_{j} \|_{C^{0}(\bar{\Omega})}^{p} .
$$

A similar estimate can be done for $\Omega_{j}^{-}$. Therefore

$$
\left\|u_{j}-v_{j}\right\|_{L^{p}(\Omega)} \leq C \varepsilon_{j}^{1 / p}|| u_{j}-v_{j} \|_{C^{0}(\bar{\Omega})} .
$$


From Lemma 6.4, we have

$$
\left\|u_{j}-v_{j}\right\|_{C^{0}(\bar{\Omega})} \leq C\left\|u_{j}-v_{j}\right\|_{W_{\varepsilon}^{2, p}(\Omega)} .
$$

Finally, by Lemma 6.5 we have

$$
\left\|u_{j}-v_{j}\right\|_{W_{\varepsilon}^{2, p}(\Omega)} \leq C\left\|u_{j}-v_{j}\right\|_{L^{p}(\Omega)} .
$$

Combining our all estimates, we get

$$
\left\|u_{j}-v_{j}\right\|_{L^{p}(\Omega)} \leq C \varepsilon_{j}^{1 / p}\left\|u_{j}-v_{j}\right\|_{L^{p}(\Omega)},
$$

which is true only when $u_{j}-v_{j} \equiv 0$. This completes the proof of Case 1 .

For Case 2 , we extend $u_{j}$ evenly to a larger rectangle $(0,2 a) \times(-b, b)$, and we still use $u_{j}$ to denote the extended solutions. Then $\mathcal{N}\left(u_{j}\right)$ consists of two curves which meet at $(a, 0)$. Since $u_{j}$ satisfies the equation

$$
\varepsilon_{j}^{2} \Delta\left(u_{j}-\alpha\right)+h_{j}(\mathbf{x})\left(u_{j}-\alpha\right)=0, \quad \mathbf{x} \in \Omega
$$

where

$$
h_{j}(\mathbf{x})= \begin{cases}\frac{f\left(u_{j}(\mathbf{x})\right)}{u_{j}(\mathbf{x})-\alpha} & u_{j}(\mathbf{x}) \neq \alpha \\ f^{\prime}\left(u_{j}(\mathbf{x})\right) & \text { otherwise }\end{cases}
$$

then by Cheng [C], page 49, Theorem 2.5, the nodal lines $\mathcal{N}\left(u_{j}\right)$ form an equiangular system. Since $u_{j}$ is extended evenly, then each component of $\mathcal{N}\left(u_{j}\right)$ forms a $45^{\circ}$ angle with the neighboring edges. Note that in the result of Cheng $[\mathrm{C}]$, it is assumed that $h \in C^{\infty}(O)$, where $O$ is a neighborhood of the critical point of nodal lines. However the proof there also works for $h \in C^{1}$, which is satisfied in our case when $f$ is $C^{2}$.

Away from the vertex point, $\mathcal{N}\left(u_{j}\right)$ is nearly a straight line, by the same proof as in Step 1 of Case 1 , so $\mathcal{N}\left(u_{j}\right)$ is approximately a line with slope -1 in $\Omega$. If the other end of $N\left(u_{j}\right)$ is not a vertex, we reach a contradiction since $\mathcal{N}\left(u_{j}\right)$ must be orthogonal to the interior of an edge. Thus the other end must be the upper left vertex, and in particular we must have $a=b$. This completes the proof of Case 2, and also the theorem.

\section{Remark 6.7.}

1. In Theorem 6.6, we conclude that the only possible solution such that $u_{x}<0$ and $u_{y}<0$ in $\Omega$ when $\lambda$ is large is a solution with a diagonal nodal line. In S4, we prove that such solutions do exist, and if we extend these solutions to $(0,2 a) \times(-a, a)$, then as $\lambda \rightarrow \infty$, the extended solutions (after a rescaling) converge to a solution of (1.7), and

$$
\begin{aligned}
& \alpha<U(x, y)<M, \text { if }|x|>|y|, \\
& m<U(x, y)<\alpha, \quad \text { if }|x|<|y|, \\
& U(x, y)=\alpha, \quad \text { if }|x|=|y| .
\end{aligned}
$$

An entire solution of (1.7) satisfying these properties is called a saddle solution, following Dang, Fife and Peletier [DFP]. For related discussions on this aspect, we refer to S4. 
2. The techniques in the proof of Theorem 6.6 should be useful for more general situations. In particular, the proof of Step 1 can also be used to prove: Let $(\lambda, u)$ be a solution of (1.4) with a smooth bounded convex domain $\Omega \subset \mathbf{R}^{2}$ and a balanced $f$. If a connected component $K$ of the nodal set $\mathcal{N}(u)$ is a simple curve, then $K$ is nearly a straight line which intersects $\partial \Omega$ orthogonally.

\section{REFERENCES}

[ABF] Alikakos, Nicholas; Bates, Peter W.; Fusco, Giorgio, Slow motion for the Cahn-Hilliard equation in one space dimension. J. Differential Equations, 90, (1991), no. 1, 81-135. MR 92a:35152

$[\mathrm{ABrF}] \quad$ Alikakos, N. D.; Bronsard, L.; Fusco, G., Slow motion in the gradient theory of phase transitions via energy and spectrum. Calc. Var. Partial Differential Equations, 6, (1998), no. 1, 39-66. MR 99d:82025

[AF1] Alikakos, Nicholas D.; Fusco, Giorgio, Slow dynamics for the Cahn-Hilliard equation in higher space dimensions. I. Spectral estimates. Comm. Partial Differential Equations, 19, (1994), no. 9-10, 1397-1447. MR 95j:35163

[AF2] Alikakos, Nicholas D.; Fusco, Giorgio, Slow dynamics for the Cahn-Hilliard equation in higher space dimensions: the motion of bubbles. Arch. Rational Mech. Anal., 141, (1998), no. 1, 1-61. MR 99d:35013

[AFK] Alikakos, Nicholas D.; Fusco, Giorgio; Kowalczyk, Michal, Finite-dimensional dynamics and interfaces intersecting the boundary: equilibria and quasi-invariant manifold. Indiana Univ. Math. J., 45, (1996), no. 4, 1119-1155. MR 98b:35077

[AFS] Alikakos, Nicholas D.; Fusco, Giorgio; Stefanopoulos, Vagelis, Critical spectrum and stability of interfaces for a class of reaction-diffusion equations. J. Differential Equations, 126, (1996), no. 1, 106-167. MR 97d:35100

[BDS] Bates, Peter W.; Dancer, Norman E.; Shi, Junping, Multi-spike stationary solutions of the Cahn-Hilliard equation in higher-dimensional and instability. Advances of Differential Equations, 4, (1999), no. 1, 1-69. MR 99k:35097

[BFi] Bates, Peter W.; Fife, Paul C., The dynamics of nucleation for the Cahn-Hilliard equation. SIAM J. Appl. Math., 53, (1993), no. 4, 990-1008. MR 94g:82034

[BFu Bates, Peter W.; Fusco, Giorgio, Equilibria with many nuclei for the Cahn-Hilliard equation. J. Differential Equations, 160, (2000), no. 2, 283-356. MR 2001b:35045

[BS] Bates, Peter W.; Shi, Junping, Existence and instability of spike layer solutions to singular perturbation problems. To appear in J. Funct. Anal., (2002).

[BX1] Bates, Peter W.; Xun, Jianping, Metastable patterns for the Cahn-Hilliard equation. I. J. Differential Equations 111 (1994), no. 2, 421-457. MR 95e:35172

[BX2] Bates, Peter W.; Xun, Jianping, Metastable patterns for the Cahn-Hilliard equation. II. J. Differential Equations 117 (1995), no. 1, 165-216. MR 95m:35188

[BCN] Berestycki, Henri; Caffarelli, Luis; Nirenberg, Louis, Further qualitative properties for elliptic equations in unbounded domains. Dedicated to Ennio De Giorgi. Ann. Scuola Norm. Sup. Pisa Cl. Sci. (4), 25, (1997), no. 1-2, 69-94. MR 2000e:35053

[BK] Bronsard, Lia; Kohn, Robert V., On the slowness of phase boundary motion in one space dimension. Comm. Pure Appl. Math., 43, (1990), no. 8, 983-997. MR 91f:35023

$[\mathrm{BrF}]$ Brunovský, Pavol; Fiedler, Bernold, Numbers of zeros on invariant manifolds in reaction-diffusion equations. Nonlinear Anal., 10, (1986), no. 2, 179-193. MR 88c:35080

[CGS] Carr, Jack; Gurtin, Morton E.; Slemrod, Marshall, Structured phase transitions on a finite interval. Arch. Rational Mech. Anal., 86, (1984), no. 4, 317-351. MR 86i:80001

[CP] Carr, J.; Pego, R. L., Metastable patterns in solutions of $u_{t}=\epsilon^{2} u_{x x}-f(u)$. Comm. Pure Appl. Math., 42, (1989), no. 5, 523-576. MR 90f:35091

[CaH] Casten, Richard G.; Holland, Charles J., Instability results for reaction diffusion equations with Neumann boundary conditions. J. Differential Equations, 27, (1978), no. 2, 266-273. MR 80a:35064

[CI] Chafee, N.; Infante, E. F., A bifurcation problem for a nonlinear partial differential equation of parabolic type. Applicable Anal., 4, (1974/75), 17-37. MR 55:13084

[C] Cheng, Shiu Yuen, Eigenfunctions and nodal sets. Comment. Math. Helv., 51, (1976), no. 1, 43-55. MR 53:1661 
[CR] Crandall, Michael G.; Rabinowitz, Paul H, Bifurcation from simple eigenvalues. J. Functional Analysis, 8, (1971), 321-340. MR 44:5836

[D1] Dancer, E. N., On the number of positive solutions of weakly nonlinear elliptic equations when a parameter is large. Proc. London Math. Soc. (3), 53, (1986), no. 3, 429-452. MR 88c:35061

[D2] Dancer, E. N., On the uniqueness of the positive solution of a singularly perturbed problem. Rocky Mountain J. Math., 25, (1995), no. 3, 957-975. MR 96j:35021

[D3] Dancer, E. N., On positive solutions of some singularly perturbed problems where the nonlinearity changes sign. Topol. Methods Nonlinear Anal., 5, (1995), no. 1, 141-175. MR 96i:35037

[DY1] Dancer, E. N.; Yan, Shusen, Multipeak solutions for a singularly perturbed Neumann problem. Pacific J. Math., 189, (1999), no. 2, 241-262. MR 2000d:35010

[DY2] Dancer, E. N.; Yan, Shusen, A singularly perturbed elliptic problem in bounded domains with nontrivial topology. Adv. Differential Equations, 4, (1999), no. 3, 347-368. MR 2000d:35009

[DFP] Dang, Ha; Fife, Paul C.; Peletier, L. A., Saddle solutions of the bistable diffusion equation. Z. Angew. Math. Phys., 43, (1992), no. 6, 984-998. MR 94b:35041

[FKMW] Fife, Paul C.; Kielhöfer, Hansjörg; Maier-Paape, Stanislaus; Wanner, Thomas, Perturbation of doubly periodic solution branches with applications to the Cahn-Hilliard equation. Phys. D., 100, (1997), no. 3-4, 257-278. MR 98a:35004

[FH1] Fusco, G.; Hale, J. K. Stable equilibria in a scalar parabolic equation with variable diffusion. SIAM J. Math. Anal., 16, (1985), no. 6, 1152-1164. MR 87c:35078

[FH2] Fusco, G.; Hale, J. K., Slow-motion manifolds, dormant instability, and singular perturbations. J. Dynamics Differential Equations, 1, (1989), no. 1, 75-94. MR 90i:35131

[GT] Gilbarg, David; Trudinger, Neil S., Elliptic partial differential equations of second order. Second edition. Springer-Verlag, Berlin-New York, (1983). MR 86c:35035

[GG] Ghoussoub, N.; Gui, C., On a conjecture of De Giorgi and some related problems. Math. Ann., 311 (1998), no. 3, 481-491. MR 99j:35049

[G] Gui, Changfeng, Multipeak solutions for a semilinear Neumann problem. Duke Math. J., 84, (1996), no. 3, 739-769. MR 97i:35052

[GW1] Gui, Changfeng; Wei, Juncheng, Multiple interior peak solutions for some singularly perturbed Neumann problems. J. Differential Equations, 158, (1999), no. 1, 1-27. MR 2000g:35035

[GW2] Gui, Changfeng; Wei, Juncheng, On multiple mixed interior and boundary peak solutions for some singularly perturbed Neumann problems. Canad. J. Math., 52, (2000), no. 3, 522-538. MR 2001b:35023

[GWW] Gui, Changfeng; Wei, Juncheng; Winter, Matthias, Multiple boundary peak solutions for some singularly perturbed Neumann problems. Ann. Inst. H. Poincaré Anal. Non Linéaire, 17, (2000), no. 1, 47-82. MR 2001a:35018

[HK] Healey, Timothy J.; Kielhöfer, H, Symmetry and nodal properties in the global bifurcation analysis of quasi-linear elliptic equations. Arch. Rational Mech. Anal., 113, (1990), no. 4, 299-311. MR 92d:35042

$[\mathrm{H}] \quad$ Henry, Daniel, Geometric theory of semilinear parabolic equations. Lecture Notes in Mathematics, 840. Springer-Verlag, Berlin-New York, (1981). MR 83j:35084

[Ki] Kielhöfer, Hansjörg, Pattern formation of the stationary Cahn-Hilliard model. Proc. Roy. Soc. Edinburgh Sect. A, 127, (1997), no. 6, 1219-1243. MR 99b:35055

[KS] Kohn, Robert V.; Sternberg, Peter, Local minimisers and singular perturbations. Proc. Roy. Soc. Edinburgh Sect. A, 111 (1989), no. 1-2, 69-84. MR 90c:49021

[Kor] Korman, Philip, Exact multiplicity of solutions for a class of semilinear Neumann problems. Preprint, (2000).

[Kow] Kowalczyk, Michal, Multiple spike layers in the shadow Gierer-Meinhardt system: existence of equilibria and the quasi-invariant manifold. Duke Math. J., 98, (1999), no. 1, 59-111. MR 2000d:35073

[L] Li, Yanyan, On a singular perturbed equation with Neumann boundary condition. Comm. Partial Differential Equations, 23, (1998), no. 3-4, 487-545. MR 2000a:35013

[LNT] Lin, C.-S.; Ni, W.-M.; Takagi, I., Large amplitude stationary solutions to a chemotaxis system. J. Differential Equations, 72, (1988), no. 1, 1-27. MR 89e:35075 
[M1] Matano, Hiroshi, Asymptotic behavior and stability of solutions of semilinear diffusion equations. Publ. Res. Inst. Math. Sci., 15, (1979), no. 2, 401-454. MR 80m:35046

[M2] Matano, Hiroshi, Nonincrease of the lap-number of a solution for a one-dimensional semilinear parabolic equation. J. Fac. Sci. Univ. Tokyo Sect. IA Math., 29, (1982), no. 2, 401-441. MR 84m:35060

[MM] Maier-Paape, Stanislaus; Miller, Ulrich, The set of equilibria for the Allen-Cahn equation on the square. Preprint, (2000).

[Me] Mei, Zhen, Solution branches of a semilinear elliptic problem at corank-2 bifurcation points with Neumann boundary conditions. Proc. Roy. Soc. Edinburgh Sect. A, 123, (1993), no. 3, 479-495. MR 94j:35025

[MS] Mizoguchi, Noriko; Suzuki, Takashi, Equations of gas combustion: S-shaped bifurcation and mushrooms. J. Differential Equations, 134, (1997), no. 2, 183-215. MR 97m:35090

[N] Ni, Wei-Ming, Diffusion, cross-diffusion, and their spike-layer steady states. Notices Amer. Math. Soc., 45, (1998), no. 1, 9-18. MR 99a:35132

[NT1] Ni, Wei-Ming; Takagi, Izumi, On the Neumann problem for some semilinear elliptic equations and systems of activator-inhibitor type. Trans. Amer. Math. Soc., 297, (1986), no. 1, 351-368. MR 87k:35091

[NT2] Ni, Wei-Ming; Takagi, Izumi, On the shape of least-energy solutions to a semilinear Neumann problem. Comm. Pure Appl. Math., 44, (1991), no. 7, 819-851. MR 92i:35052

[NT3] Ni, Wei-Ming; Takagi, Izumi, Locating the peaks of least-energy solutions to a semilinear Neumann problem. Duke Math. J., 70, (1993), no. 2, 247-281. MR 94h:35072

[OS1] Ouyang Tiancheng; Shi, Junping, Exact multiplicity of positive solutions for a class of semilinear problem. Jour. Differential Equations, 146, no. 1, 121-156, (1998). MR 99f:35061

[OS2] Ouyang Tiancheng; Shi, Junping, Exact multiplicity of positive solutions for a class of semilinear problems: II. Jour. Differential Equations, 158, no. 1, 94-151, (1999). MR 2001b:35117

[PT] Padilla, Pablo; Tonegawa, Yoshihiro, On the convergence of stable phase transitions. Comm. Pure Appl. Math., 51, (1998), no. 6, 551-579. MR 99b:58054

[R] Rabinowitz, Paul H., Some global results for nonlinear eigenvalue problems. J. Func. Anal., 7, (1971), 487-513. MR 46:745

[Sc] Schaaf, Renate, Global solution branches of two-point boundary value problems. Lecture Notes in Mathematics, 1458. Springer-Verlag, Berlin, (1990). MR 92a:34003

[S1] Shi, Junping, Persistence and bifurcation of degenerate solutions. Jour. Func. Anal., 169, no. 2, 494-531, (1999). MR 2001h:47115

[S2] Shi, Junping, Exact multiplicity of solutions to superlinear and sublinear problems. To appear in Nonlinear Anal., (2001).

[S3] Shi, Junping, Blow-up points of solution curves for a semilinear problem. Topological Meth. of Nonlinear Anal., 15, no. 2, 251-266, (2000). MR 2002b:35060

[S4] Shi, Junping, Saddle solutions of the balanced bistable diffusion equation. To appear in Comm. Pure Appl. Math., (2002).

[S5] Shi, Junping, Entire solutions and solutions to singularly perturbed semilinear problems with unbalanced nonlinearities. In preparation.

[SS] Shi, Junping; Shivaji, Ratnasingham, Global bifurcation for concave semipositon problems. To appear in Recent Advances in Evolution Equations, (2002).

[SWj] Shi, Junping; Wang, Junping, Morse indices and exact multiplicity of solutions to semilinear elliptic problems. Proc. of Amer. Math. Soc., 127, (1999), no. 12, 36853695. MR 2000d:35082

[SmW] Smoller, J.; Wasserman, A., Global bifurcation of steady-state solutions. J. Differential Equations, 39, (1981), no. 2, 269-290; errata, ibid. 77, (1989), 199-202. MR 82d:58056. MR 90f:58136

[T] Taniguchi, Masaharu, Bifurcation from flat-layered solutions to reaction diffusion systems in two space dimensions. J. Math. Sci. Univ. Tokyo, 1, (1994), no. 2, 339-367. MR 96b:35109

[TN] Taniguchi, Masaharu; Nishiura, Yasumasa, Instability of planar interfaces in reactiondiffusion systems. SIAM J. Math. Anal. 25, (1994), no. 1, 99-134. MR 94b:35029

[W] Wei, Juncheng, On the boundary spike layer solutions to a singularly perturbed Neumann problem. J. Differential Equations, 134, (1997), no. 1, 104-133. MR 98e:35076 
[WW1] Wei, Juncheng; Winter, Matthias, Stationary solutions for the Cahn-Hilliard equation. Ann. Inst. H. Poincaré Anal. Non Linéaire, 15, (1998), no. 4, 459-492. MR 2000b:35093

[WW2] Wei, Juncheng; Winter, Matthias, Multi-peak solutions for a wide class of singular perturbation problems. J. London Math. Soc., 59, (1999), no. 2, 585-606. MR 2000h:35012

Department of Mathematics, College of William and Mary, Williamsburg, Virginia 23187, and Department of Mathematics, Harbin Normal University, Harbin, HeilongJIANG, P. R. ChINA 150080

E-mail address: shij@math.wm.edu

$U R L:$ http://www.math.wm.edu/ shij/ 